\title{
Geometric approach to nonvariational singular elliptic equations
}

\author{
Damião Araújo \& Eduardo V. Teixeira \\ $\star$ Final Version $\star$ \\ $C^{2}$ regularity assumption on $F$-harmonic functions removed
}

\begin{abstract}
In this work we develop a systematic geometric approach to study fully nonlinear elliptic equations with singular absorption terms as well as their related free boundary problems. The magnitude of the singularity is measured by a negative parameter $(\gamma-1)$, for $0<\gamma<1$, which reflects on lack of smoothness for an existing solution along the singular interface between its positive and zero phases. We establish existence as well sharp regularity properties of solutions. We further prove that minimal solutions are non-degenerate and obtain fine geometricmeasure properties of the free boundary $\mathfrak{F}=\partial\{u>0\}$. In particular we show sharp Hausdorff estimates which imply local finiteness of the perimeter of the region $\{u>0\}$ and $\mathscr{H}^{n-1}$ a.e. weak differentiability property of $\mathfrak{F}$.
\end{abstract}

\section{Introduction}

The aim of this present work is to study fine qualitative properties of nonvariational singular elliptic equations of the form

$$
\left\{\begin{aligned}
F\left(D^{2} u\right) & \sim u^{-\theta} \cdot \chi_{\{u>0\}} & & \text { in } \\
u & =f & & \text { on } \partial \Omega
\end{aligned}\right.
$$

where $\Omega \subset \mathbb{R}^{N}$ is a smooth bounded domain, $\theta=1-\gamma$, for $0<\gamma<1, f$ is a positive, $C^{2}$ boundary datum and the governing operator $F$ is assumed to be uniform elliptic, i.e., $\left(D_{(i, j)} F\right)_{1 \leq i, j \leq N}$ is a positive definite matrix. The study of singular equations as (1.1) is motivated by applications in a number of problems in engineering sciences. In fact the free boundary problem

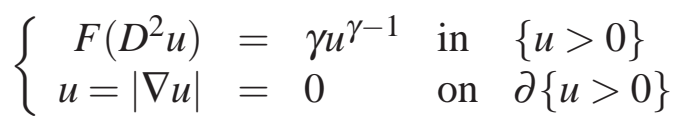

is used, for example, to model fluids passing through a porous body $\Omega$. For instance, $u$ could represent the density of a gas, or else the density of certain chemical specie, in reaction with a porous catalyst pellet, $\Omega$. 
The variational theory, $F(M)=\operatorname{Tr}(M)$, for the free boundary problem (1.2) is fairly well understood, nowadays. It appears as the Euler-Lagrange equation in the minimization of nondifferentiable functionals:

$$
\int \frac{1}{2}|\nabla u(X)|^{2}+u(X)^{\gamma} d X \longrightarrow \min
$$

See, for instance [21, 22, 2, 27]. Notice that such a problem is quite different from the one treated in the classical paper [11]. The latter has been recently studied in the fully nonlinear setting in [13].

The case $\gamma=1$ in (1.3) represents the obstacle problem, [6]; the case $\gamma=0$ relates to the cavitation problem, [1]. Fully nonlinear version of the obstacle problem has been considered in [19]. Nonvariational cavitation problem has been recently studied in [23]. The delicate intermediary case, $0<\gamma<1$, addressed in this present work brings major novelty adversities as the equation satisfied within the positive set $\{u>0\}$ is nonhomogeneous and blows-up along the a priori unknown quenching interface $\mathfrak{F}=\partial\{u>0\} \cap \Omega$ - the so called free boundary of the problem. The lack of variational or energy approaches too implies significant difficulties in the problem and new, nonvariational solutions have to be established. In fact, since the free boundary problem considered in this paper has nonvariational character, one cannot use the powerful measure-distributional language to setup weak version of the problem. Instead we shall employ a perturbation scheme and will obtain uniform estimates with respect to the approximating parameter $\varepsilon$. A solution to the fully nonlinear free boundary problem (1.2) will therefore be obtained as the limit of appropriate approximating configurations.

The first main problem to be addressed concerns the optimal regularity for solutions to Equation (1.1). Optimal estimates for heterogeneous equations, $\mathrm{L} u=f(X, u)$ is in general a quite delicate issue. For the singular setting studied in this present work, optimal estimates are even more involved as they can be understood as invariant (tangential) equations for their own scaling. We show in Section 4 of the present work that solutions are locally of class $C^{1, \frac{\gamma}{2-\gamma}}$ at the free boundary. This result was only known in the variational setting, for minimizers of Euler-Lagrange functional, see [21, 22, 2] and [14, 15].

The next principal result devilered in this article states that minimal solutions, i.e., solutions obtained from Perron's type method do grow precisely as $\operatorname{dist}(X, \mathfrak{F})^{1+\frac{\gamma}{2-\gamma}}$, which corresponds to the maximum growth rate allowed. Such a result implies a quite restrictive geometry for the free quenching interface $\mathfrak{F}$. As consequence of our sharp gradient estimate, Theorem 4.2 and optimal growth rate, Theorem 5.4, a minimal solution is trapped between the graph of two multiples of $\operatorname{dist}(X, \mathfrak{F})^{1+\frac{\gamma}{2-\gamma}}$, i.e.,

$$
\underline{c} \cdot \operatorname{dist}(X, \mathfrak{F})^{1+\frac{\gamma}{2-\gamma}} \leq u(X) \leq \bar{C} \cdot \operatorname{dist}(X, \mathfrak{F})^{1+\frac{\gamma}{2-\gamma}}, \quad X \in\{u>0\}
$$

By means of geometric considerations, in Section 6 we establish a clean Harnack inequality for solutions to (1.1) within free boundary tangential balls, $B \subset\{u>0\}, B$ tangent to $\mathfrak{F}$. In Section 7 under an extra asymptotic structural assumption on the governing operator $F$, we establish Hausdorff estimates of the free boundary. In particular we show $\chi_{\{u>0\} \cap \Omega^{\prime}} \in \mathrm{BV}(\Omega)$, that is, 
$\{u>0\}$ is locally a set of finite perimeter. We further show that the reduced free boundary has $\mathscr{H}^{n-1}$ total measure. The last two Sections close up the project by obtaining a solution to the fully nonlinear free boundary problem (1.2) with the desired analytic and geometric properties.

\section{Mathematical set-up}

Throughout this paper $\Omega$ will be a fixed Lipschitz bounded domain in $\mathbb{R}^{N}, f: \partial \Omega \rightarrow \mathbb{R}_{+}$is a continuous boundary datum and $0<\gamma<1$ is a fixed real number. We shall denote by $\operatorname{Sym}(N)$ the space of all real $N \times N$ symmetric matrices and $F$ will be a uniformly elliptic fully nonlinear operator; that is, we shall assume that there exist two constants $0<\lambda \leq \Lambda$ such that

$$
F(\mathscr{M}+\mathscr{N}) \leq F(\mathscr{M})+\Lambda\left\|\mathscr{N}^{+}\right\|-\lambda\left\|\mathscr{N}^{-}\right\|, \quad \forall \mathscr{M}, \mathscr{N} \in \operatorname{Sym}(N)
$$

The ultimate goal of this paper is to study existence and fine qualitative properties of solutions to the singular equation

$$
F\left(D^{2} u\right)=\gamma u^{\gamma-1} \cdot \chi_{\{u>0\}}
$$

From the equation itself, one notices that the Hessian of an existing solution blows-up along the free boundary $\mathfrak{F}=\partial\{u>0\} \cap \Omega$; therefore, solutions cannot be of class $C^{2}$. In the fully nonlinear setting, the problem of optimal regularity for solutions to Equation (2.2) is a rather delicate issue and it will be addressed in Section 4, Part of the subtleness of this problem comes from the intrinsic complexity of the regularity theory for viscosity solutions to uniform elliptic equations. We recall that it is well known that solutions to homogeneous equation

$$
F\left(D^{2} u\right)=0
$$

has a priori $C^{1, \mu}$ bounds for some $\mu>0$ that depends only on $N, \lambda$ and $\Lambda$. Under concavity or convexity assumption on $F$, a Theorem due to Evans and Krylov, states that solutions are $C^{2, \alpha}$. Nevertheless, Nadirashvili and Vladut have recently shown that given any $0<\eta<1$ it is possible to build up a uniformly elliptic operator $F$, whose solutions to the homogeneous equation (2.3) are not $C^{1, \eta}$, see [20], Theorem 1.1.

Let us turn our attention to the singularly perturbed strategy we shall use in order to grapple with the lack of variational approaches available. In this paper we suggest the following singular perturbation scheme to appropriately approach the free boundary problem (1.2):

$$
\left\{\begin{aligned}
F\left(D^{2} u\right) & =\beta_{\varepsilon}(u), & & \text { in } \\
u & =f & & \text { on } \partial \Omega
\end{aligned}\right.
$$

The singular perturbation term $\beta_{\varepsilon}$ is build up as follows: initially select your favorite function $\rho \in C_{0}^{\infty}[0,1]$ and set

$$
\alpha:=1+\frac{\gamma}{2-\gamma} .
$$


Throughout the whole paper, $\alpha$ will always be the fixed value stated in (2.4). In the sequel, define

$$
B_{\varepsilon}(t)=\int_{0}^{\frac{t-\varepsilon^{\alpha} \sigma_{0}}{\varepsilon^{\alpha}}} \rho(s) d s,
$$

where $0<\sigma_{0}<\frac{1}{2}$ is an arbitrary technical choice. Notice that $B_{\varepsilon}$ is a smooth approximation of $\chi_{(0, \infty)}$. Finally, we set

$$
\beta_{\varepsilon}(t)=\gamma t^{\gamma-1} B_{\varepsilon}(t)
$$

Such a construction is carefully carried out as to preserve the natural scaling of the desired equation (2.2).

We finish this Section by listing the main notations adopted throughout the article:

- The dimension of the Euclidean space the problem is modeled in will be denoted by $N \geq 2$. $\Omega$ will be a fixed bounded domain in $\mathbb{R}^{N}$. For a domain $\mathscr{O} \subset \mathbb{R}^{N}, \partial \mathscr{O}$ will represent the boundary of the domain $\mathscr{O} . \chi_{S}$ will stand for the characteristic function of the set $S$.

- The $N$-dimensional Lebesgue measure of a set $A \subset \mathbb{R}^{N}$ will be denoted by $\mathcal{L}^{N}(A)$. $\mathscr{H}^{n-1}$ will stand for the $(n-1)$-Hausdorff measure.

- $\langle\cdot, \cdot\rangle$ will be the standard scalar product in $\mathbb{R}^{N}$. For a vector $\xi=\left(\xi_{1}, \cdots, \xi_{N}\right) \in \mathbb{R}^{N}$, its Euclidean norm will be denoted by $|\xi|:=\sqrt{\langle\xi, \xi\rangle}$. The tensor product $\xi \otimes \psi$ denotes the matrix whose entries are given by $\xi_{i} \psi_{j}$ for $1 \leq i, j \leq N$.

- $B_{r}(p)$ will be the open ball centered at $p$ with radius $r$. Furthermore, we shall denote $k B=$ $k B_{r}(p):=B_{k r}(p)$, for any $k>0$.

- $\operatorname{Spect}(\mathscr{M})$ denotes the set of eigenvalues of the matrix $\mathscr{M}$.

- Fixed the ellipticity constants $0<\lambda \leq \Lambda, \mathcal{P}^{-}(M)$ and $\mathcal{P}^{+}(M)$ denote the Pucci extremal operators:

$$
\begin{aligned}
& \mathcal{P}^{-}(M):=\inf \{\operatorname{Tr}(A \cdot M) \mid \lambda \mathrm{Id} \leq A \leq \Lambda \mathrm{Id}\} \\
& \mathcal{P}^{+}(M):=\sup \{\operatorname{Tr}(A \cdot M) \mid \lambda \mathrm{Id} \leq A \leq \Lambda \mathrm{Id}\}
\end{aligned}
$$

- Constants $C, C_{1}, C_{2}, \cdots>0$ and $c, c_{0}, c_{1}, c_{2}, \cdots>0$ that depend only on dimension, $\gamma$ and ellipticity constants $\lambda, \Lambda$ will be call universal. Any additional dependence will be emphasized.

\section{Existence of minimal solutions}

In this section we comment on the existence of a viscosity solution to equation $\left(E_{\varepsilon}\right)$. More importantly, we shall establish herein a stable process to select special solutions to $\left.E_{\varepsilon}\right]$. As we will show in Section 5, the family of minimal solutions turns out to satisfy the desired appropriate geometric 
features. Such properties will allow us to establish Hausdorff estimates of the free boundary in Section 7

Notice that because of the lack of monotonicity of equation $\left(E_{\varepsilon}\right)$ with respect to the variable $u$, classical Perron's method cannot be directly employed. The next theorem proved in [23], is an adaptation of Perron's method, which is by now fairly well understood.

Theorem 3.1. Let $g$ be a bounded, Lipschitz function defined in the real line $\mathbb{R}$. Suppose F uniformly elliptic and that the equation $F\left(D^{2} u\right)=g(u)$ admits a Lipschitz viscosity subsolution $u_{\star}$ and a Lipschitz viscosity supersolution $u^{\star}$ such that $u_{\star}=u^{\star}=f \in C(\partial \Omega)$. Define the set of functions,

$$
S:=\left\{w \in C(\bar{\Omega}) ; \quad u_{\star} \leq w \leq u^{\star} \quad \text { and } w \text { supersolution of } F\left(D^{2} u\right)=g(u)\right\} .
$$

Then,

$$
v(x):=\inf _{w \in S} w(x)
$$

is a continuous viscosity solution of $F\left(D^{2} u\right)=g(u)$ and $v=f$ continuously on $\partial \Omega$.

Existence of minimal solution to Equation $\left(E_{\varepsilon}\right)$ follows by choosing $u_{\star}=u_{\star}(\varepsilon)$ and $u^{\star}=u^{\star}(\varepsilon)$ solutions to the following boundary value problems

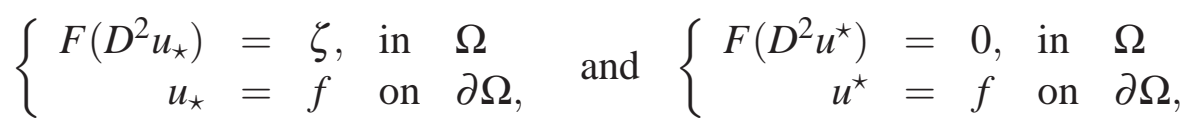

where

$$
\zeta:=\sup \beta_{\varepsilon} \sim \varepsilon^{\gamma-1}
$$

The existence the functions $u_{\star}$ and $u^{\star}$ is consequence of standard Perron's method. By construction $u_{\star}$ is viscosity subsolution of $\left(E_{\varepsilon}\right)$ and $u^{\star}$ is a viscosity supersolution of $\left(E_{\varepsilon}\right)$. Note that $u^{\star}, u_{\star} \in$ $C^{0,1}(\Omega) \cap C(\bar{\Omega})$. Thus a direct application of Theorem 3.1 yields the following existence result:

Theorem 3.2 (Existence of minimal solutions). Let $\Omega \in \mathbb{R}^{n}$ be a Lipschitz domain and $f \in C(\partial \Omega)$ be a nonnegative boundary datum. Then, for each $\varepsilon>0$ fixed, equation $\left(E_{\varepsilon}\right)$ has a nonnegative minimal viscosity solution $u_{\varepsilon} \in C(\bar{\Omega})$.

As previously mentioned, more importantly than assuring existence of a viscosity solution to $\left(E_{\varepsilon}\right)$, Theorem 3.2 provides a particular choice of solutions to such an equation. In comparison with the variational theory, this choice is a replacement for the selection of minimizers of the Euler-Lagrange functional (see for instance [25] for further details). Therefore, unless otherwise stated, whenever we mention viscosity solution to $\left(E_{\varepsilon}\right)$, we mean the minimal solution provided by Theorem 3.2

\section{Sharp regularity estimates}

The first main result we prove in this paper is the optimal regularity estimate, uniform in $\varepsilon$, available for solutions to $\left(E_{\varepsilon}\right)$. We will show that $u_{\varepsilon}$ is locally a $C^{1, \beta}$ function and we shall further 
determine the optimal $\beta>0$ in terms of the degree of singularity $\gamma$. This key information has only been known for variational solutions, [21, 14, 15] and the proofs make decisive use of energy considerations. In principle it is not even clear that one should expect the same regularity theory for nonvariational problems.

Thus, we start off this Section by rather informal, heuristic considerations as to guide us through the genuine results to be established later on. Let us analyze the limiting free boundary problem (1.2). Suppose 0 is a free boundary point and, say, $-\mathrm{e}_{n}$ is the unit outward normal pointing towards the quenching phase $\{u=0\}$. If $u$ is $C^{1, \beta}$ at 0 , then, in a small neighborhood, say, $B_{\rho} \cap\{u>0\}, \rho \ll 1, u$ behaves like $\sim X_{n}^{1+\beta}$. Therefore, the singular potential of the equation in (1.2) is like $\sim X_{n}^{(1+\beta) \cdot(1-\gamma)}$. In view of the regularity theory for heterogeneous fully nonlinear equations $F\left(D^{2} u\right)=f(X)$, established in [7] and [26], we obtain the following implication

$$
X_{n}^{(1+\beta) \cdot(1-\gamma)} \in L_{\mathrm{weak}}^{\theta} \quad \text { implies } \quad u \in C^{1,1-\frac{1}{\theta}} .
$$

The reasoning above gives the following system of algebraic equations

$$
\left\{\begin{aligned}
\theta(1+\beta)(\gamma-1) & =-1 \\
\beta & =1-\frac{1}{\theta} .
\end{aligned}\right.
$$

Solving for $\beta$, revels, $\beta=\frac{\gamma}{2-\gamma}$, which agrees with the optimal regularity estimate established for the variational theory.

This Section is devoted to establish local $C^{1, \frac{\gamma}{2-\gamma}}$ regularity estimates for solutions $u_{\varepsilon}$ to Equation $\left(E_{\varepsilon}\right)$, uniform in $\varepsilon$ at free boundary points. In fact we shall obtain a universal growth control on $u_{\varepsilon}$ near the free boundary. The desired regularity along the free interface will then follow.

Hereafter, let us fix a point $X_{0} \in \Omega$ and for simplicity take $X_{0}=0$. Our analysis will be based on the auxiliary function $v_{\varepsilon}$, defined by

$$
v_{\varepsilon}(X):=u_{\varepsilon}^{\frac{2-\gamma}{2}}(X)
$$

For the sake of notation convenience, let us omit the subscript $\varepsilon$ in $v_{\varepsilon}$ and in $u_{\varepsilon}$, writing $\operatorname{simply} v$ and $u$ to denote these functions. Formally one computes

$$
\begin{aligned}
\nabla v(X) & =\left(1-\frac{\gamma}{2}\right) u^{\frac{-\gamma}{2}}(X) \cdot \nabla u(X) \\
D^{2} v(X) & =-\frac{\gamma}{2}\left(1-\frac{\gamma}{2}\right) u^{-1-\frac{\gamma}{2}}(X) \cdot \nabla u(X) \otimes \nabla u(X)+\left(1-\frac{\gamma}{2}\right) u^{\frac{-\gamma}{2}}(X) \cdot D^{2} u(X)
\end{aligned}
$$

Plugging (4.2) into (4.3) yields

$$
D^{2} v(X)=-\left(\frac{\gamma}{2-\gamma}\right) v^{-1}(X) \cdot \nabla v(X) \otimes \nabla v(X)+\left(1-\frac{\gamma}{2}\right) u^{1-\gamma}(X) \cdot D^{2} u(X) \cdot v^{-1}(X) .
$$

From the PDE satisfied by $u$, we have

$$
\begin{aligned}
u^{1-\gamma}(X) v^{-1}(X) \cdot F\left(D^{2} u\right) & =\gamma\left(u^{1-\gamma} \cdot \beta_{\varepsilon}(u)\right) \cdot v^{-1}(X) \\
& =\gamma B_{\varepsilon}\left(v^{\alpha}\right) \cdot v^{-1}(X)
\end{aligned}
$$


Thus, ellipticity and (4.4) yield

$$
F\left(D^{2} v(X)+\frac{\gamma}{2-\gamma} v^{-1}(X) \cdot \nabla v(X) \otimes \nabla v(X)\right)=f(X) v^{-1}(X),
$$

for a bounded function $f(X)$. Though the above computation has been conducted formally, it is standard to justify Equation (4.7) using the language of viscosity solutions.

Our first result towards optimal regularity establishes equicontinuity for functions satisfying (4.7), which implies the same conclusion to the family of functions $u_{\varepsilon}$. The proof is an adaptation of the Ishii-Lions method [17], see also [3], [4] and [16].

Proposition 4.1 ( $C^{0}$-compactness). Solutions to (4.7) are universally locally uniform continuous, that is, there exists a universal modulus of continuity, $\rho$, such that $\left|v\left(X_{1}\right)-v\left(X_{2}\right)\right| \leq C_{\Omega^{\prime}} \rho\left(\mid X_{1}-\right.$ $\left.X_{2}\right)$ ), for $X_{1}, X_{2} \in \Omega^{\prime} \Subset \Omega$.

Proof. Fixed $X_{0} \in \Omega$, let us denote by $r=\operatorname{dist}\left(X_{0}, \partial \Omega\right)$. We will show that for any $\delta>0$ given, there exists $L_{\delta}>0$ such that

$$
\Phi:=\sup _{(X, Y) \in \bar{\Omega} \times \bar{\Omega}}\left\{v(X)-v(Y)-L_{\delta} \omega(|X-Y|)-\frac{8\|v\|_{\infty}}{r^{2}}\left(\left|X-X_{0}\right|^{2}+\left|Y-X_{0}\right|^{2}\right)\right\} \leq \delta,
$$

where $\omega(t)=t-\frac{1}{10 \sqrt{r}} t^{3 / 2}$, for $t \leq r, \omega(t)=\frac{9}{10} r$ for $t \geq r$. For that, suppose $\Phi>\delta$ and let $(\bar{X}, \bar{Y})$ be a maximum point. It readily follows that

$$
\frac{8\|v\|_{\infty}}{r^{2}}\left(\left|\bar{X}-X_{0}\right|^{2}+\left|\bar{Y}-X_{0}\right|^{2}\right)<2\|v\|_{\infty}
$$

thus, $\bar{X}$ and $\bar{Y}$ are interior points and $|\bar{X}-\bar{Y}|<r$. Clearly $\bar{X} \neq \bar{Y}$, otherwise $\Phi=0<\delta$. Define in the sequel the vectors

$$
\begin{aligned}
& \xi_{X}:=L_{\delta} \omega^{\prime}(|\bar{X}-\bar{Y}|) \eta+\frac{16\|v\|_{\infty}}{r^{2}}\left(\bar{X}-X_{0}\right) \\
& \xi_{Y}:=L_{\delta} \omega^{\prime}(|\bar{X}-\bar{Y}|) \eta-\frac{16\|v\|_{\infty}}{r^{2}}\left(\bar{Y}-X_{0}\right),
\end{aligned}
$$

where $\eta:=\frac{\bar{X}-\bar{Y}}{|\bar{X}-\bar{Y}|}$. From Jensen-Ishii's approximation Lemma, see [10] and also [4], for $\imath>0$ small enough, it is possible to find matrices $M_{X}$ and $M_{Y}$ with

$$
\begin{aligned}
& \left(\xi_{X}, M_{X}\right) \in J^{-}(v, \bar{X}), \\
& \left(\xi_{Y}, M_{Y}\right) \in J^{+}(v, \bar{Y}),
\end{aligned}
$$

where $J^{-}$and $J^{+}$denote the subjet and superjet respectively (see [10] for definition), verifying the following matrix inequality

$$
\left(\begin{array}{cc}
M_{X} & 0 \\
0 & -M_{Y}
\end{array}\right) \leq\left(\begin{array}{cc}
Z & -Z \\
-Z & Z
\end{array}\right)+\left(\frac{16\|v\|_{\infty}}{r^{2}}+\imath\right) \operatorname{Id}_{2 n \times 2 n},
$$


where

$$
Z=\omega^{\prime \prime}(|\bar{X}-\bar{Y}|) \frac{(\bar{X}-\bar{Y}) \otimes(\bar{X}-\bar{Y})}{|\bar{X}-\bar{Y}|^{2}}+\frac{\omega^{\prime}(|\bar{X}-\bar{Y}|)}{|\bar{X}-\bar{Y}|}\left\{\operatorname{Id}_{n \times n}-\frac{(\bar{X}-\bar{Y}) \otimes(\bar{X}-\bar{Y})}{|\bar{X}-\bar{Y}|^{2}}\right\} .
$$

Applying inequality (4.14) to vectors of the form $(\xi, \xi)$, we conclude

$$
\operatorname{Spect}\left(M_{Y}-M_{X}\right) \in\left(-\frac{32\|v\|_{\infty}}{r^{2}}-\imath,+\infty\right) .
$$

However, if we apply to the special vector $(\eta,-\eta)$, we conclude

$$
\operatorname{Spect}\left(M_{Y}-M_{X}\right) \cap\left(\frac{c}{\sqrt{r}} L_{\delta}-\frac{32\|v\|_{\infty}}{r^{2}}-\imath+\infty\right) \neq \emptyset,
$$

for a universal number $c>0$, easily computed if one desires.

In the sequel we use Equation (4.7), together with (4.12) and (4.13) to write up the following ponitwise inequalities

$$
\begin{aligned}
& F\left(M_{X}+\frac{\gamma}{2-\gamma} v^{-1}(\bar{X}) \cdot \xi_{X} \otimes \xi_{X}\right) \geq f(\bar{X}) v^{-1}(\bar{X}) \\
& F\left(M_{Y}+\frac{\gamma}{2-\gamma} v^{-1}(\bar{Y}) \cdot \xi_{Y} \otimes \xi_{Y}\right) \leq f(\bar{Y}) v^{-1}(\bar{Y}) .
\end{aligned}
$$

Subtracting (4.18) from (4.19) and using ellipticity, we find

$$
\mathcal{P}^{-}\left(\left[M_{Y}-M_{X}\right]+\frac{\gamma}{2-\gamma}\left[v^{-1}(\bar{Y}) \cdot \xi_{Y} \otimes \xi_{Y}-v^{-1}(\bar{X}) \cdot \xi_{X} \otimes \xi_{X}\right]\right) \leq f(\bar{Y}) v^{-1}(\bar{Y})-f(\bar{X}) v^{-1}(\bar{X}),
$$

where $\mathcal{P}^{-}$is the extremal Pucci operator with ellipticity $(\lambda, \Lambda)$. If we label $\Xi:=\left[M_{Y}-M_{X}\right]+$ $\frac{\gamma}{2-\gamma}\left[v^{-1}(\bar{Y}) \cdot \xi_{Y} \otimes \xi_{Y}-v^{-1}(\bar{X}) \cdot \xi_{X} \otimes \xi_{X}\right]$, from the definition of $\mathcal{P}^{-}$, there exists a $(\lambda, \Lambda)$-elliptic matrix, $\lambda \mathrm{Id} \leq a_{i j} \leq \Lambda \mathrm{Id}$, satisfying

$$
\operatorname{Tr}\left(a_{i j} \Xi_{i j}\right)-1 \leq \mathcal{P}^{-}(\Xi) .
$$

From (4.16), (4.17) and ellipticity, we estimate

$$
\operatorname{Tr}\left(a_{i j}\left(M_{Y}-M_{X}\right)_{i j}\right) \geq \lambda \frac{c}{\sqrt{r}} L_{\delta}-n \Lambda\left(\frac{32\|v\|_{\infty}}{r^{2}}-\imath\right) .
$$

Now we compute

$$
\operatorname{Tr}\left(a_{i j}\left(\xi_{Y} \otimes \xi_{Y}\right)_{i j}\right)=\left(\frac{17}{20} L_{\delta}\right)^{2} \cdot \operatorname{Tr}\left(a_{i j} \eta_{i} \eta_{j}\right)-\frac{256\|v\|_{\infty}^{2}}{r^{4}} \cdot \operatorname{Tr}\left(a_{i j}\left(\bar{Y}-X_{0}\right)_{i}\left(\bar{Y}-X_{0}\right)_{j}\right),
$$

and likewise

$$
\operatorname{Tr}\left(a_{i j}\left(\xi_{X} \otimes \xi_{X}\right)_{i j}\right)=\left(\frac{17}{20} L_{\delta}\right)^{2} \cdot \operatorname{Tr}\left(a_{i j} \eta_{i} \eta_{j}\right)+\frac{256\|v\|_{\infty}^{2}}{r^{4}} \cdot \operatorname{Tr}\left(a_{i j}\left(\bar{X}-X_{0}\right)_{i}\left(\bar{X}-X_{0}\right)_{j}\right)
$$


From ellipticity follows the estimates

$$
\begin{aligned}
\sigma:=\operatorname{Tr}\left(a_{i j} \eta_{i} \eta_{j}\right) & \geq \lambda, \\
\max \left\{\operatorname{Tr}\left(a_{i j}\left(\bar{Y}-X_{0}\right)_{i}\left(\bar{Y}-X_{0}\right)_{j}\right), \operatorname{Tr}\left(a_{i j}\left(\bar{X}-X_{0}\right)_{i}\left(\bar{X}-X_{0}\right)_{j}\right)\right\} & \leq \Lambda r^{2} .
\end{aligned}
$$

Also, since $v(\bar{X})-v(\bar{Y})>\delta$, we readily have

$$
\begin{aligned}
v^{-1}(\bar{X}) & <\frac{1}{\delta} \\
v^{-1}(\bar{Y}) & >\frac{\delta}{\|v\|_{\infty}^{2}}+v^{-1}(\bar{X}) .
\end{aligned}
$$

Combining (4.20), (4.21), (4.22), (4.23) and (4.24), if $L_{\delta} \gg 1$, depending only on universal numbers, and $r$, we obtain,

$$
\begin{aligned}
0 & <\lambda \frac{c}{\sqrt{r}} L_{\delta}-n \Lambda\left(\frac{32\|v\|_{\infty}}{r^{2}}-\imath\right)-1 \\
& \leq\left[\frac{\gamma}{2-\gamma}\left(\frac{17}{20} L_{\delta}\right)^{2} \cdot \sigma+\frac{\gamma}{2-\gamma} \cdot \frac{256 \Lambda\|v\|_{\infty}^{2}}{r^{2}}-f(\bar{X})\right] v^{-1}(\bar{X}) \\
& -\left[\frac{\gamma}{2-\gamma}\left(\frac{17}{20} L_{\delta}\right)^{2} \cdot \sigma-\frac{\gamma}{2-\gamma} \cdot \frac{256 \Lambda\|v\|_{\infty}^{2}}{r^{2}}-f(\bar{Y})\right] v^{-1}(\bar{Y})
\end{aligned}
$$

Now, we select $L_{\delta}$ even bigger, depending further on $\gamma,\|v\|_{\infty},\|f\|_{\infty}$ so that

$$
\frac{\frac{\gamma}{2-\gamma}\left(\frac{17}{20} L_{\delta}\right)^{2} \cdot \sigma+\frac{\gamma}{2-\gamma} \cdot \frac{256 \Lambda\|v\|_{\infty}^{2}}{r^{2}}-f(\bar{X})}{\frac{\gamma}{2-\gamma}\left(\frac{17}{20} L_{\delta}\right)^{2} \cdot \sigma-\frac{\gamma}{2-\gamma} \cdot \frac{256 \Lambda\|v\|_{\infty}^{2}}{r^{2}}-f(\bar{Y})}<\left(1+\frac{\delta^{2}}{\|v\|_{\infty}^{2}}\right) .
$$

Estimates (4.28), (4.29) and (4.30) give

$$
\frac{\delta}{\|v\|_{\infty}^{2}}+v^{-1}(\bar{X})<v^{-1}(\bar{Y})<\left(1+\frac{\delta^{2}}{\|v\|_{\infty}^{2}}\right) v^{-1}(\bar{X}) .
$$

Finally, confronting (4.31) and (4.27) we end up with

$$
\frac{\delta}{\|v\|_{\infty}^{2}}<\frac{\delta^{2}}{\|v\|_{\infty}^{2}} v^{-1}(\bar{X})<\frac{\delta}{\|v\|_{\infty}^{2}},
$$

which is a contradiction. Thus, if $L_{\delta} \gg 1$, indeed $\Phi<\delta$ and the proof of Proposition 4.1 follows.

An inference from the proof above reveals that in fact $L_{\delta} \sim \delta^{-1}$, as to attain (4.30). Thus, Proposition 4.1 gives local $C^{0, \frac{1}{2}}$ continuity for $v$. We further comment that the proof could also be performed using $\omega(t)=t^{\theta}$, with $0<\theta<1$, as $|X-Y|^{\theta}$ too is concave in the radial direction (information used to obtain (4.17)). We shall use this observation later.

Theorem 4.2 (Uniform optimal regularity). Given a subset $\Omega^{\prime} \Subset \Omega$, there exists a constant $C$ depending on, $\|f\|_{\infty}, \gamma, \Omega^{\prime}$, dimension, ellipticity, but independent of $\varepsilon$, such that, any family of viscosity solutions $\left\{u_{\varepsilon}\right\}$ of equation $\left(E_{\varepsilon}\right)$ satisfies,

$$
\sup _{B_{r}(X)} u_{\varepsilon} \leq C\left(r^{\frac{2}{2-\gamma}}+u_{\varepsilon}(X)\right), \quad \forall X \in \Omega^{\prime} .
$$


Proof. Suppose, for the sake of contradiction, the thesis of Theorem 4.2 fails to hold. Combining discrete iterative techniques and a continuous methods, see [9], Lemma 3.3 and also [24] for similar reasoning, for each $k>1$, it is possible to find $0<r_{k}=\mathrm{o}(1), X_{k} \in \Omega^{\prime}, \varepsilon_{k}>0$ such that the following two inequalities hold

$$
\begin{aligned}
s_{k}:=\sup _{B_{r_{k}}\left(X_{k}\right)} u_{\varepsilon_{k}}>k\left(r_{k}^{\alpha}+u_{\varepsilon_{k}}\left(X_{k}\right)\right) \\
\sup _{B_{r_{k}}\left(X_{k}\right)}\left[u_{\varepsilon_{k}}-u_{\varepsilon_{k}}\left(X_{k}\right)\right] \geq 2^{-\alpha k} \sup _{B_{2^{k_{r_{k}}}}\left(X_{k}\right)}\left[u_{\varepsilon_{k}}-u_{\varepsilon_{k}}\left(X_{k}\right)\right] .
\end{aligned}
$$

The normalized function $\varphi_{k}: B_{1} \rightarrow \mathbb{R}$ given by

$$
\varphi_{k}(Y):=\frac{u_{\varepsilon_{k}}\left(X_{k}+r_{k} Y\right)}{s_{k}},
$$

satisfies

$$
\begin{aligned}
0 \leq \varphi_{k}(Y) & \leq C|Y|^{\alpha} \\
\varphi_{k}(0) & =\mathrm{o}(1), \\
\sup _{B_{1}} \varphi_{k} & =1 .
\end{aligned}
$$

In addition, the following equation is satisfied in the viscosity sense

$$
F_{k}\left(D^{2} \varphi_{k}(Y)\right)=\left(\frac{r_{k}^{\alpha}}{s_{k}}\right)^{\frac{2}{\alpha}} \beta_{\varepsilon_{k}}\left(\varphi_{k}\right)
$$

where

$$
F_{k}(\mathscr{M}):=\frac{r_{k}^{2}}{s_{k}} \cdot F\left(\frac{s_{k}}{r_{k}^{2}} \mathscr{M}\right),
$$

which is a $(\lambda, \Lambda)$-elliptic operator. Thus, from Proposition 4.1, up to a subsequence, $\varphi_{k}$ converges locally uniformly to an entire function $\varphi_{0}: \mathbb{R}^{N} \rightarrow \mathbb{R}$. From hypothesis of contradiction (4.34),

$$
\left(\frac{r_{k}^{\alpha}}{s_{k}}\right)^{\frac{2}{\alpha}}=\mathrm{o}(1)
$$

Passing to another subsequence, if necessary, $F_{k}$ converges locally uniformly to a limiting recession operator $\tilde{F}$, which is $(\lambda, \Lambda)$-elliptic and homogeneous of degree one for nonnegative scalars. Passing the limit as $k \rightarrow \infty$ in (4.40) yields

$$
\varphi_{0}^{1-\gamma} \cdot \tilde{F}\left(D^{2} \varphi_{0}(Y)\right)=0
$$

Notice, in view of (4.38) and (4.39), we have

$$
\varphi_{0}(0)=0, \quad \sup _{B_{1}} \varphi_{0}=1 .
$$


We now revisit the proof of Proposition 4.1, Defining $\psi:=\varphi_{0}^{1 / \alpha}$, we find

$$
\tilde{F}\left(D^{2} \psi+c_{\gamma} \psi^{-1} \nabla \psi \otimes \nabla \psi\right)=0 .
$$

By running the same reasonings of the proof of Proposition 4.1, for $\psi$, with $\omega=t^{\theta}, 0<\theta<1$, $\delta=0, f=0$ and with no localization term, gives $C^{0, \theta}$ estimates for $\psi$, for any $\theta<1$. In fact, for $L \gg 1$, depending only on ellipticity, estimate (4.29) becomes

$$
0<c L^{2} \cdot\left(\psi^{-1}(\bar{X})-\psi^{-1}(\bar{Y})\right)<0,
$$

since the contradiction assumption in the reasoning of the proof of Proposition 4.1 implies $\psi(\bar{X})>$ $\psi(\bar{Y})$. We now choose $\theta_{0}$ so that

$$
\frac{1}{\alpha}<\theta_{0}<1
$$

A final contradiction is then obtained when we confront (4.44) with the $C^{0, \theta_{0}}$ regularity for $\psi$. Indeed, select a point $Z$ in $\left\{\varphi_{0}>0\right\}$ and $Z_{0} \in\left\{\varphi_{0}=0\right\}$, satisfying $\operatorname{dist}\left(Z,\left\{\varphi_{0}=0\right\}\right)=\left|Z-Z_{0}\right|<\frac{1}{2}$. It follows from Hopf maximum principle that

$$
0<\liminf _{h \rightarrow 0} \frac{\varphi\left(h e+Z_{0}\right)}{h},
$$

where $e$ is the inward normal vector to the ball $B_{\left|Z-Z_{0}\right|}(Z)$ at $Z_{0}$. On the other hand, we have

$$
\begin{aligned}
\frac{\varphi_{0}\left(h e+Z_{0}\right)}{|h|} & =\sqrt[\theta_{0}]{\frac{\varphi_{0}^{\frac{1}{\alpha}}\left(h e+Z_{0}\right)}{|h|^{\theta_{0}}}} \cdot \varphi_{0}^{1-\frac{1}{\alpha \theta_{0}}}\left(h e+Z_{0}\right) \\
& \leq C \cdot \varphi_{0}^{\delta_{0}}\left(h e+Z_{0}\right) \\
& \rightarrow 0
\end{aligned}
$$

as $h \rightarrow 0$. This concludes the proof of Theorem 4.2 .

\section{Nondegeneracy of minimal solutions}

In the previous Section we have shown that solutions to Equation $\left(E_{\varepsilon}\right)$ are locally of class $C^{1, \frac{\gamma}{2-\gamma}}$. In particular such an estimate provides an upper bound on how fast $u_{\varepsilon}$ growths away from, say, the level surface $\left\{u_{\varepsilon} \sim \varepsilon^{\alpha}\right\}$, for $\alpha$ as in (2.4). That is,

$$
u_{\varepsilon}(Z) \lesssim\left[\operatorname{dist}\left(Z,\left\{u_{\varepsilon} \sim \varepsilon^{\alpha}\right\}\right)\right]^{\alpha} .
$$

The main result we shall prove in this Section states that minimal solutions do growth precisely as $\operatorname{dist}\left(X_{0},\left\{u_{\varepsilon} \sim \varepsilon^{\alpha}\right\}\right)^{\alpha}$, see Corollary 5.5 for the precise statement. In fact we shall establish a 
stronger nondegeneracy property of minimal solutions, which also has fundamental importance in our blow-up analysis.

To simplify the statement of the results, we introduce some definitions and notations. Hereafter we shall use systematically we following notations:

$$
\begin{aligned}
\left\{u_{\varepsilon}>\kappa\right\} & :=\left\{x \in \Omega \mid u_{\varepsilon}(x)>\kappa\right\} \\
\left\{\tau>u_{\varepsilon}>\lambda\right\} & :=\left\{x \in \Omega \mid \tau>u_{\varepsilon}(x)>\lambda\right\} \\
d_{\varepsilon}(X) & :=\operatorname{dist}\left(X, \partial\left\{u_{\varepsilon}>\varepsilon^{\alpha}\right\}\right)
\end{aligned}
$$

The nondegeneracy feature of minimal solutions is based on the construction of appropriate viscosity supersolution whose value within an inter disk is much smaller than its value on the boundary of an outer disk.

Proposition 5.1. Assume, with no loss of generality that $0 \in \Omega$. Given $0<\eta$, there exists a radially symmetric function $\theta \in C^{1,1}(\Omega)$ and universal small constants $0<c_{2}<1$ and $0<c_{1}<1$ such that

1. $\theta \equiv 2 \sigma_{0}$ in $B_{c_{1}} \eta$

2. $\theta \geq c_{2} \eta^{1+\frac{\gamma}{2-\gamma}}$ in $\Omega \backslash B_{\eta}$

3. $\theta$ is satisfies $F\left(D^{2} \theta(X)\right) \leq \beta(\theta(X))$, pointwise in $\Omega$, where $\beta=\beta_{1}$, as in (2.6).

Proof. Initially define

$$
\theta(X)= \begin{cases}2 \sigma_{0} & \text { for } \quad 0 \leq|X| \leq c_{1} \eta \\ a_{0}\left(|X|-c_{1} \eta\right)^{2}+2 \sigma_{0} & \text { for } c_{1} \eta \leq|X| \leq \eta \\ A|X|^{\alpha}+B & \text { for } \quad|X| \geq \eta\end{cases}
$$

where the constants $a_{0}, A, B$ e $c_{1}$ will be chosen later. Our first goal is to enforce that such a function is indeed $C^{1,1}$. For this, we have to set along $|X|=\eta$,

$$
a_{0}\left(1-c_{1}\right)^{2} \eta^{2}+2 \sigma_{0}=\theta(X)=A \eta^{\alpha}+B
$$

thus, easily we obtain

$$
a_{0}=\frac{1}{\left(1-c_{1}\right)^{2}}\left[A \eta^{\alpha-2}+\eta^{-2}\left(B-2 \sigma_{0}\right)\right] .
$$

Moreover, differentiating $\theta$ and matching its gradient along $|X|=\eta$, we obtain

$$
2 a_{0}\left(1-c_{1}\right) X_{i}=A \alpha \eta^{\alpha-2} X_{i}
$$

Combining (5.1) and (5.2) we find

$$
\frac{A \alpha \eta^{\alpha-2}}{2\left(1-c_{1}\right)}=\frac{1}{\left(1-c_{1}\right)^{2}}\left[A \eta^{\alpha-2}+\eta^{-2}\left(B-2 \sigma_{0}\right)\right] .
$$

In the sequel, take

$$
c_{1}:=\frac{\gamma}{2} \in(0,1)
$$


which implies the relation $\alpha=\frac{1}{1-c_{1}}$, where, as always, $\alpha$ is the value set in (2.4) . Finally we set

$$
B=2 \sigma_{0}-\frac{A}{2} \eta^{\alpha}
$$

as to (5.3) to be satisfied. Summing up the construction so far, we have built up

$$
\theta(X)= \begin{cases}2 \sigma_{0} & \text { for } \quad 0 \leq|X| \leq c_{1} \eta \\ \frac{A \alpha^{2}}{2} \eta^{\alpha-2}\left(|X|-c_{1} \eta\right)^{2}+2 \sigma_{0} & \text { for } \quad c_{1} \eta \leq|X| \leq \eta \\ A|X|^{\alpha}+\left(2 \sigma_{0}-\frac{A}{2} \eta^{\alpha}\right) & \text { for } \quad|X| \geq \eta\end{cases}
$$

which is of $C^{1,1}$, by the construction itself. We still have the parameter $A$ to be adjusted later. Our next step is to show that $\theta$ is an appropriate supersolution, that is, we want to establish

$$
F\left(D^{2} \theta\right) \leq \beta(\theta)
$$

pointwise. To this end, we first analyze the equation in the region $c_{1} \eta \leq|X| \leq \eta$. Direct computations yield

$$
\theta_{i j}=A \alpha^{2} \eta^{\alpha-2}\left[\frac{X_{i} X_{j}}{|X|^{2}}+\left(1-\frac{c_{1} \eta}{|X|}\right)\left(\delta_{i j}-\frac{X_{i} X_{j}}{|X|^{2}}\right)\right],
$$

within $c_{1} \eta \leq|X| \leq \eta$. At a point of the form $\bar{X}=(|X|, 0, \cdots, 0)$, we find out

$$
\begin{aligned}
& \theta_{11}=A \alpha^{2} \eta^{\alpha-2} \\
& \theta_{i i}=A \alpha^{2} \eta^{\alpha-2}\left(1-\frac{c_{1} \eta}{|X|}\right) \text { if } i>1 \\
& \theta_{i j}=0 \quad \text { if } i \neq j .
\end{aligned}
$$

By symmetric invariance of $\theta$ and ellipticity of $F$, we obtain

$$
F\left(D^{2} \theta(X)\right) \leq \Lambda\left[A \alpha^{2} \eta^{\alpha-2}+(N-1) A \alpha^{2} \eta^{\alpha-2}\left(1-\frac{c_{1} \eta}{|X|}\right)\right] \leq \Lambda N A \alpha^{2} \eta^{\alpha-2}
$$

Recall $N$ is the dimension of the space. However, within the region $c_{1} \eta \leq|X| \leq \eta$, we have

$$
2 \sigma_{0} \leq \theta(X) \leq \frac{A}{2} \eta^{\alpha}+2 \sigma_{0}
$$

Taking into account that the function $B=B_{1}$ set in (2.5) is non-decreasing, we readily obtain

$$
\begin{aligned}
\beta(\theta(X)) & \geq \gamma \theta(X)^{\gamma-1} B\left(2 \sigma_{0}\right) \\
& \geq \gamma \theta(\eta)^{\gamma-1} B\left(2 \sigma_{0}\right) \\
& \geq \gamma\left(\frac{A}{2} \eta^{\alpha}+2 \sigma_{0}\right)^{\gamma-1} B\left(2 \sigma_{0}\right)
\end{aligned}
$$


Therefore, taking $0<A \ll 1$ small enough,

$$
\gamma\left(\frac{A}{2} \alpha \eta^{\alpha}+2 \sigma_{0}\right)^{\gamma-1} B\left(2 \sigma_{0}\right)>\frac{1}{2} \gamma\left(2 \sigma_{0}\right)^{\gamma-1} B\left(2 \sigma_{0}\right)>\Lambda N A \alpha^{2} \eta^{\alpha-2} .
$$

and we indeed obtain the desired pointwise inequality

$$
F\left(D^{2} \theta\right) \leq \beta(\theta(X)),
$$

in the region $c_{1} \eta \leq|X| \leq \eta$. Let us turn our attention to the region $\eta \leq|X|$. Readily we have

$$
\theta_{i j}=A \alpha\left[(\alpha-2)|X|^{\alpha-4} X_{i} X_{j}+\delta_{i j}|X|^{\alpha-2}\right] \text {. }
$$

Thus, at a point of the form $(|X|, 0, \cdots, 0)$, we obtain

$$
\begin{aligned}
\theta_{11} & =A \alpha(\alpha-1)|X|^{\alpha-2} & & \\
\theta_{i i} & =A \alpha|X|^{\alpha-2} & & \text { if } i>1 \\
\theta_{i j} & =0 & & \text { if } i \neq j .
\end{aligned}
$$

Therefore, again by symmetric invariance of $\theta$ and ellipticity of $F$, we can write

$$
F\left(D^{2} \theta(X)\right) \leq \Lambda[A \alpha(\alpha-1)+(N-1) A \alpha]|X|^{\alpha-2} \leq \Lambda N A \alpha \eta^{\alpha-2} .
$$

On the other hand, in the region $\eta \leq|X|$, we have for $M \geq \sup _{X \in \Omega}|X|$, that

$$
M^{\alpha} \geq|X|^{\alpha}-\frac{\eta^{\alpha}}{2}>0
$$

and so,

$$
\beta(\theta(X)) \geq \gamma\left(A\left(|X|^{\alpha}-\frac{1}{2} \eta^{\alpha}\right)+2 \sigma_{0}\right)^{\gamma-1} B(\theta(\eta))>\gamma\left(A M^{\alpha}+2 \sigma_{0}\right)^{\gamma-1} B(\theta(\eta)) .
$$

Thus, adjusting $A>0$ even smaller, if necessary, we can assure

$$
A M^{\alpha}+2 \sigma_{0}<4 \sigma_{0}
$$

and therefore,

$$
\beta(\theta(X))>\gamma\left(4 \sigma_{0}\right)^{\gamma-1} B(\theta(\eta)) .
$$

Finally by 5.6 and the inequality above, as well as diminishing the value of $A>0$ even further, if necessary, we reach

$$
F\left(D^{2} \theta(X)\right) \leq \Lambda N A \alpha|X|^{\alpha-2}<\gamma\left(4 \sigma_{0}\right)^{\gamma-1} B(\theta(\eta)) \leq \beta(\theta(X))
$$

So its follow (3). By construction (2) is valid, and the proof of Proposition 5.1 follows. 
Proposition 5.1 provides the existence of the appropriate barrier in the unit scale $\varepsilon=1$. To furnish the desired supersolution for any $\varepsilon>0$ small we argue as follows. Fixed $\varepsilon>0$, we consider the fully nonlinear elliptic operator

$$
F_{\varepsilon}(\mathscr{M}):=\varepsilon^{2-\alpha} F\left(\varepsilon^{\alpha-2} \mathscr{M}\right) .
$$

It is standard to verify that $F_{\varepsilon}$ is uniform elliptic with the same ellipticity constants as $F$. Proposition 5.1 applied to $F_{\varepsilon}$ provides a $C^{1,1}$ function $\theta=\theta(\varepsilon)$ that satisfies the differential inequality

$$
F_{\varepsilon}\left(D^{2} \theta(X)\right)=\varepsilon^{2-\alpha} F\left(\varepsilon^{\alpha-2} D^{2} \theta(X)\right) \leq \beta_{1}(\theta(X)) .
$$

Finally, we define

$$
\theta_{\varepsilon}(X):=\varepsilon^{\alpha} \theta\left(\varepsilon^{-1} X\right)
$$

where once more, $\alpha$ is the value set in (2.4). We verify readily that $\theta_{\varepsilon}$ defined above satisfies

$$
\begin{aligned}
& \checkmark \quad \theta_{\varepsilon}=2 \sigma_{0} \varepsilon^{\alpha} \text { in } B_{c_{1} \varepsilon \eta} ; \\
& \checkmark \quad \theta_{\varepsilon} \geq c_{2} \eta^{\alpha} \text { in } \Omega \backslash B_{\varepsilon \eta} ; \\
& \checkmark \quad \theta_{\varepsilon} \in C^{1,1}(\Omega) \text { and it is a supersolution to }\left(E_{\varepsilon}\right) .
\end{aligned}
$$

We are ready to establish strong nondegeneracy of minimal solutions to the singularly perturbed problem $\left(E_{\varepsilon}\right)$.

Theorem 5.2 (Strong nondegeneracy). Let $X_{0} \in\left\{u_{\varepsilon}>\varepsilon^{\alpha}\right\}$. There exist two universal positive constants $c_{0}>0$ and $r_{0}>0$ such that if $r<r_{0}$, there holds

$$
\sup _{B_{r}\left(X_{0}\right)} u_{\varepsilon} \geq c_{0} r^{\alpha}
$$

for $\alpha$ as in (2.4).

Proof. Given $r<r_{0}$, we construct $\theta_{\varepsilon}$ for $\eta=r / \varepsilon$. By minimality of $u_{\varepsilon}$,

$$
u_{\varepsilon}(Z)>\theta_{\varepsilon}(Z)
$$

for some point $Z \in \partial B_{r}\left(X_{0}\right)$. Indeed, suppose for the sake of contradiction that $u_{\varepsilon} \leq \theta_{\varepsilon}$ along $\partial B_{r}$. Define

$$
w_{\varepsilon}=\left\{\begin{array}{cl}
\min \left\{\theta_{\varepsilon}, u_{\varepsilon}\right\} & \text { in } \overline{B_{r}} ; \\
u_{\varepsilon} & \text { in } \Omega \backslash \overline{B_{r}} .
\end{array}\right.
$$

Thus, $w_{\varepsilon}$ is supersolution to $E_{\varepsilon}$; however in $B_{c_{1} r}$, we have,

$$
u_{\varepsilon}>\varepsilon^{\alpha}>2 \sigma_{0} \varepsilon^{\alpha} \equiv \theta_{\varepsilon}=w_{\varepsilon},
$$

which contradicts the minimality of $u_{\varepsilon}$. In conclusion,

$$
c_{2} r^{\alpha} \leq \theta_{\varepsilon}(Z)<u_{\varepsilon}(Z) \leq \sup _{B_{r}} u_{\varepsilon}
$$

and the Theorem is proven. 
An immediate Corollary of Theorem 5.2 combined with Corollary 4.33 is the upper and lower control of $u_{\varepsilon}$ by $r^{\alpha}$ in $B_{r} \subset\left\{u_{\varepsilon}>\varepsilon^{\alpha}\right\}$.

Corollary 5.3. Given a subdomain $\Omega^{\prime} \Subset \Omega$, there exists a universal constant $C=C\left(\Omega^{\prime}\right)$ such that for $X_{0} \in \Omega^{\prime} \cap\left\{u_{\varepsilon}>\varepsilon^{\alpha}\right\}$ and $r \leq r_{0}$,

$$
C^{-1} r^{\alpha} \leq \sup _{B_{r}\left(X_{0}\right)} u_{\varepsilon} \leq C\left(r^{\alpha}+u_{\varepsilon}\left(X_{0}\right)\right)
$$

Recall we have set the following notation: $d_{\varepsilon}(X)=\operatorname{dist}\left(X, \partial\left\{u_{\varepsilon}>\varepsilon^{\alpha}\right\}\right)$. Our next step is to show that in fact $u_{\varepsilon}$ does growth at the sharp rate away from the free boundary, that is $\sim d_{\varepsilon}^{\alpha}$.

Theorem 5.4 (Sharp Growth). Let $X_{0} \in\left\{u_{\varepsilon}>\varepsilon^{\alpha}\right\}$. Then there exists $c_{0}>0$ universal such that

$$
u_{\varepsilon}\left(X_{0}\right) \geq c_{0} d_{\varepsilon}\left(X_{0}\right)^{\alpha}
$$

Proof. Let us suppose for sake of contradiction that no such a constant exists. If so, there would exist a sequence of points $X_{n} \in\left\{u_{\varepsilon}>\varepsilon^{\alpha}\right\}$, with $d_{n}:=d_{\varepsilon}\left(X_{n}\right) \rightarrow 0$ and

$$
u_{\varepsilon}\left(X_{n}\right) \leq \frac{1}{n} d_{n}^{\alpha}
$$

Let us define

$$
v_{n}(Y):=\frac{1}{d_{n}^{\alpha}} u_{\varepsilon}\left(X_{n}+d_{n} Y\right)
$$

The function $v_{n} \geq 0$ in $B_{1}$, and easily we verify that $v_{n}$ is a minimal solution to

$$
F_{n}\left(D^{2} v_{n}\right)=\gamma v_{n}^{1-\gamma_{B}}{ }_{\frac{\varepsilon}{d_{n}}}\left(v_{n}\right) \quad \text { in } B_{1},
$$

where $F_{n}(\mathscr{M}):=d_{n}^{2-\alpha} F\left(d_{n}^{\alpha-2} \mathscr{M}\right)$ for all $\mathscr{M} \in \operatorname{Sym}(N)$ and $B_{\frac{\varepsilon}{d_{n}}}$ is the smooth approximation of $t^{+}$set up in (2.5). From its very definition, we check that

$$
B_{\frac{\varepsilon}{d_{n}}}(t)=\left\{\begin{array}{c}
0 \text { for } \quad 0 \leq t \leq \sigma_{0}\left(\frac{\varepsilon}{d_{n}}\right)^{\alpha} \\
1 \text { for } t \leq\left(1-\sigma_{0}\right) \cdot\left(\frac{\varepsilon}{d_{n}}\right)^{\alpha} .
\end{array}\right.
$$

Since $F_{n}$ is uniformly elliptic with same ellipticity constants as $F$, we can apply Theorem 5.2 to $v_{n}$ as to obtain

$$
\sup _{B_{\kappa}} v_{n} \geq c_{0} \kappa^{\alpha}
$$

for a universal constant $c_{0}$ and for any $\kappa>0$. However, by Theorem (4.2), there holds

$$
v_{n}(X) \leq C\left(\kappa^{\alpha}+v_{n}(0)\right)
$$


for a universal constant $C>0$ and for any $\kappa>0$. In particular, for $\kappa_{0} \ll 1$,

$$
v_{n}(X) \leq C\left(\frac{\sigma_{0}}{2 C} \varepsilon^{\alpha}+v_{n}(0)\right) \quad \text { in } B_{\kappa_{0}} .
$$

If we take $n \gg 1, v_{n}(0) \leq \frac{\sigma_{0}}{2 C} \varepsilon^{\alpha}$ and then

$$
v_{n}(X) \leq \sigma_{0} \varepsilon^{\alpha} \quad \text { in } B_{\kappa_{0}}
$$

In view of Equation 5.8 and (5.9), we see

$$
F_{n}\left(D^{2} v_{n}\right)=0 \quad \text { in } B_{\kappa_{0}}
$$

for $n \gg 1$. But then, by classical homogeneous Harnack inequality, see [7], and strong nondegeneracy stated in 5.10)

$$
c_{0}\left(\frac{\kappa_{0}}{2}\right)^{\alpha} \leq \sup _{B_{\frac{\kappa_{0}}{2}}} v_{n} \leq C v_{n}(0)=\mathrm{o}(1),
$$

which finally give us a contradiction.

An important consequence of Theorem 5.4 is the complete control of $u_{\varepsilon}(X)$ in terms of the $d_{\varepsilon}(X)^{\alpha}$.

Corollary 5.5. Given a subdomain $\Omega^{\prime} \Subset \Omega$, there exists a universal constant $C=C\left(\Omega^{\prime}\right)$ such that for $X \in \Omega^{\prime} \cap\left\{u_{\varepsilon}>\varepsilon^{\alpha}\right\}$ and $\varepsilon \leq d_{\varepsilon}(X)$,

$$
C d_{\varepsilon}(X)^{\alpha} \geq u_{\varepsilon}(X) \geq C^{-1} d_{\varepsilon}(X)^{\alpha} .
$$

Proof. The inequality by below is precisely the statement of Theorem 5.4. Now for $Z \in \partial\left\{u_{\varepsilon}>\right.$ $\left.\varepsilon^{\alpha}\right\}$, such that $|Z-X|=d_{\varepsilon}(X)$, it follows from Theorem 4.2

$$
u_{\varepsilon}(X) \leq \sup _{B_{d_{\varepsilon}(X)}(Z)} u_{\varepsilon} \leq C\left(d_{\varepsilon}(X)^{\alpha}+\varepsilon^{\alpha}\right) \leq C d_{\varepsilon}(X)^{\alpha}
$$

and the Corollary is proven.

As usual a fine geometric control as the one stated in Corollary 5.5 implies uniform positive density of the approximating region $\left\{u_{\varepsilon}>\varepsilon^{\alpha}\right\}$.

Corollary 5.6. Given a subdomain $\Omega^{\prime} \Subset \Omega$, there exists constant $0<c \leq 1$, depending only on $\Omega^{\prime}$ and universal parameters, such that for any $X \in \Omega^{\prime} \cap\left\{u_{\varepsilon}>\varepsilon^{\alpha}\right\}$ and $\varepsilon \ll \delta$, we have

$$
\frac{\mathcal{L}^{N}\left(B_{\delta}(X) \cap\left\{u_{\varepsilon}>\varepsilon^{\alpha}\right\}\right)}{\mathcal{L}^{N}\left(B_{\delta}\right)} \geq c .
$$


Proof. By strong non-degeneracy there exists $Y_{0} \in \overline{B_{\delta}(X)} \cap\left\{u_{\varepsilon}>\varepsilon^{\alpha}\right\}$ such that

$$
u_{\varepsilon}\left(Y_{0}\right) \geq c_{0} \delta^{\alpha}
$$

Define $d(Y):=\left|Y_{0}-Y\right|$. By Corollary 5.3,

$$
u_{\varepsilon}\left(Y_{0}\right) \leq \sup _{B_{d(Y)}(Y)} u_{\varepsilon} \leq c_{1}\left(d(Y)^{\alpha}+u_{\varepsilon}(Y)\right)
$$

and so,

$$
\frac{1}{c_{1}}\left(c_{0} \delta^{\alpha}-c_{1} d(Y)^{\alpha}\right) \leq u_{\varepsilon}(Y) .
$$

Note that, we can choose $0 \leq \tau \ll 1$ universally small such that

$$
Y \in B_{\tau \delta}\left(Y_{0}\right) \cap B_{\delta}(X) \text { and } u_{\varepsilon}(Y) \geq \varepsilon^{\alpha} .
$$

In conclusion,

$$
\mathcal{L}^{N}\left(B_{\delta}(X) \cap\left\{u_{\varepsilon}>\varepsilon^{\alpha}\right\}\right) \geq \mathcal{L}^{N}\left(B_{\delta}(X) \cap B_{\tau \delta}\left(Y_{0}\right)\right) \geq c \delta^{N}
$$

for a universal constant $c>0$.

\section{Harnack type inequalities}

It is well established that Harnack type inequalities are among the central properties of solutions to second order elliptic equations. For non-negative viscosity solutions to fully nonlinear equations with non-homogeneous right hand side,

$$
F\left(D^{2} v\right)=f(X), \quad Q_{1}
$$

Krylov-Safonov [18] and Caffarelli [7] (see also [8], Chapter 4) proved the following sharp Harnack inequality:

$$
\sup _{Q_{1 / 2}} v \leq C(n, \lambda, \Lambda)\left(\inf _{Q_{1 / 2}} v+\|f\|_{L^{n}\left(Q_{1}\right)}\right) .
$$

As mentioned in previous Sections, one of the major mathematical difficulties in dealing with singular equations as in (1.1) is the fact that right hand side blows-up near the quenching region. In particular, if one tries to interpret the singular term $\gamma u^{\gamma-1}$ as a right hand side $f(X)$ for the equation, classical Harnack inequality (6.1) gives no information near the free boundary.

The key objective of this Section is to establish, uniform-in- $\varepsilon$ clean geometric Harnack type inequalities for solutions to equation $\left(E_{\varepsilon}\right)$.

Theorem 6.1 ( $L^{1}$-Harnack inequality). Given $\Omega^{\prime} \Subset \Omega, X_{0} \in\left\{u_{\varepsilon}>\varepsilon^{\alpha}\right\} \cap \Omega^{\prime}$. Then

$$
f_{B_{\rho}\left(X_{0}\right)} u_{\varepsilon} d x \geq c \rho^{\alpha}
$$

for a universal constant $c>0$, independent of $\varepsilon$. 
Proof. From Lemma 5.2, there is a $Z \in \overline{B_{\rho}\left(X_{0}\right)} \cap\left\{u_{\varepsilon}>\varepsilon^{\alpha}\right\}$ and a $c_{0}>0$ universal, such that

$$
u_{\varepsilon}\left(Y_{0}\right) \geq c_{0} \rho^{\alpha}
$$

As in the proof of the Corollary 5.6, for $\theta \ll 1$ but universal, we obtain

$$
u_{\varepsilon}(Y) \geq C \rho^{\alpha} \quad \text { in } \quad B_{\theta \rho}\left(Y_{0}\right)
$$

Finally,

$$
f_{B_{\rho}\left(X_{0}\right)} u_{\varepsilon} d x \geq C_{N} f_{B_{\rho}\left(X_{0}\right) \cap B_{\theta \rho}(Z)} u_{\varepsilon} d x \geq C \rho^{\alpha},
$$

for $C>0$ a universal constant. Thus, the proof is concluded.

Our next Theorem is a clean Harnack inequality for ball touching the approximating free boundary $\partial\left\{u_{\varepsilon}>\varepsilon^{\alpha}\right\}$.

Theorem 6.2 (Harnack Inequality for tangential balls). Let $X_{0} \in\left\{u_{\varepsilon}>\varepsilon^{\alpha}\right\}$ and $\varepsilon \leq d:=d_{\varepsilon}\left(X_{0}\right)$. Then, there exist a universal constant $C>0$ such that

$$
\sup _{B_{\frac{d}{2}}\left(X_{0}\right)} u_{\varepsilon} \leq C \inf _{B_{\frac{d}{2}}\left(X_{0}\right)} u_{\varepsilon}
$$

Proof. Let $\xi_{0}, \xi_{1} \in \overline{B_{\frac{d}{2}}\left(X_{0}\right)}$, such that

$$
\inf _{B_{\frac{d}{2}}\left(X_{0}\right)} u_{\varepsilon}=u\left(\xi_{0}\right) \quad \text { and } \sup _{B_{\frac{d}{2}}\left(X_{0}\right)} u_{\varepsilon}=u\left(\xi_{1}\right)
$$

As $d_{\varepsilon}\left(\xi_{0}\right) \geq \frac{d}{2}$, by nondegeneracy

$$
u_{\varepsilon}\left(\xi_{0}\right) \geq C_{1} d^{\alpha}
$$

By other hand, using the corollary 5.3 , we get

$$
u_{\varepsilon}\left(\xi_{1}\right) \leq C_{2}\left(\frac{d^{\alpha}}{2}+u_{\varepsilon}\left(X_{0}\right)\right) .
$$

Taking $Y \in \partial\left\{u_{\varepsilon}>\varepsilon^{\alpha}\right\}$, where $d=\left|X_{0}-Y\right|$, we have that

$$
u_{\varepsilon}\left(X_{0}\right) \leq \sup _{B_{d}(Y)} u_{\varepsilon} \leq C_{2}\left(d^{\alpha}+\varepsilon^{\alpha}\right) \leq C_{3} d^{\alpha}
$$

So, by the three last inequalities, we obtain

$$
\sup _{B_{\frac{d}{2}}\left(X_{0}\right)} u_{\varepsilon} \leq C \inf _{B_{\frac{d}{2}}\left(X_{0}\right)} u_{\varepsilon} .
$$

for a constant $C>0$ that does not depend of $u_{\varepsilon}$ and $\varepsilon$. 


\section{Hausdorff estimates of the free boundary}

In this section, we turn our attention to uniform geometric-measure properties of $\sim \varepsilon^{\alpha}$-level surfaces of $u_{\varepsilon}$. These surfaces approximate the limiting free boundary $\mathfrak{F}:=\partial\{u>0\} \cap \Omega$, where $u$ is the desired limiting function. Through this section we shall work under the following extra structural condition on the operator $F$ :

Definition 7.1. We say a uniformly elliptic operator $F: \operatorname{Sym}(N) \rightarrow \mathbb{R}$ is asymptotically concave if there exists a positive definite matrix $\mathscr{F}=\left(f_{i j}\right)_{i j}$ and a nonnegative constant $C_{F} \geq 0$ such that

$$
f_{i j} \mathscr{M}_{i j}-F(\mathscr{M}) \geq-C_{F},
$$

for all matrix $\mathscr{M} \in \operatorname{Sym}(N)$,

Initially, let us point out that indeed hypothesis $(\mathrm{AC})$ is an asymptotic condition as $\|M\| \gg 1$, as it suffices to hold in the limit for $\|M\| \rightarrow+\infty$. It represents a sort of concavity condition at infinity of $F$. For concave operators, $C_{F}=0$. The structural condition (AC) arises from recent considerations on the recession operator

$$
F^{\star}(\mathscr{M}):=\lim _{\mu \rightarrow 0} \mu F\left(\mu^{-1} \mathscr{M}\right) .
$$

The limiting operator $F^{\star}$ should be interpreted as the tangential equation for the natural elliptic scaling on $F$. For example, for a number of elliptic operators, it is possible to verify the existence of the limit

$$
b_{i j}:=\lim _{\|\mathscr{M}\| \rightarrow \infty} F_{i j}(\mathscr{M}) .
$$

In this case, $F^{\star}(\mathscr{M})=\operatorname{Tr}\left(b_{i j} \mathscr{M}\right)$ and $(\mathrm{AC})$ is automatically satisfied. A particularly interesting example is the class of Hessian operators of the form

$$
F_{l}(M)=f_{l}\left(\lambda_{1}, \lambda_{2}, \cdots \lambda_{N}\right):=\sum_{j=1}^{N}\left(1+\lambda_{j}^{\imath}\right)^{1 / \imath}
$$

where $l$ is an odd natural number. For this family of operators, we have $F_{l}^{\star}=\Delta$ and condition (AC) is satisfied.

In [23] it is proven that the recession operator $F^{\star}$ rules the free boundary condition for fully nonlinear cavitation problems. In [24], it is established further regularity estimates of solutions to $F\left(X, D^{2} u\right)=f(X)$ via properties of the recession function.

Before continuing, let us make few remarks as to organize some systematic arguments that will appear within the next proofs.

Remark 7.2. Given $X_{0} \in\left\{u_{\varepsilon}>\varepsilon^{\alpha}\right\}$, where $u_{\varepsilon}\left(X_{0}\right)=C_{1} \varepsilon^{\alpha}$ for $C_{1}>1$, $\varepsilon \ll \rho$ and $\rho$ universally small, we have from Theorem 4.2 that in $B_{\rho}\left(X_{0}\right)$, for $\rho \ll 1$ to be adjusted soon, there holds

$$
u_{\varepsilon}^{\gamma-1} \geq C_{2}\left(\rho^{\alpha}+C_{1} \varepsilon^{\alpha}\right)^{\gamma-1} \text {. }
$$


Therefore, if $\varepsilon$ is small as to

$$
\varepsilon^{\alpha}<\frac{\rho^{\alpha}}{C_{1}}
$$

and the radius $\rho$ is also selected universally small as to

$$
2 C_{2} \rho^{\alpha} \leq \sqrt[\gamma-1]{\frac{2}{\gamma} C_{F}}
$$

we readily obtain

$$
\gamma u_{\varepsilon}^{\gamma-1} \geq 2 C_{F} \quad \text { in } B_{\rho}\left(X_{0}\right)
$$

for $C_{F}>0$ as in $(\mathrm{AC}$. Also, as

$$
\left(1-\sigma_{0}\right) \varepsilon^{\alpha}<C_{1} \varepsilon^{\alpha}
$$

we have

$$
F\left(D^{2} u_{\varepsilon}\right)=\beta_{\varepsilon}\left(u_{\varepsilon}\right)=\gamma u_{\varepsilon}^{\gamma-1}, \quad \text { in } B_{\rho}\left(X_{0}\right) .
$$

In conclusion, we obtain that $u_{\varepsilon}$ is a $f_{i j}$-subharmonic function in $B_{\rho}\left(X_{0}\right)$ for $\varepsilon \ll 1$, i.e.,

$$
f_{i j} D_{i j} u_{\varepsilon} \geq F\left(D^{2} u_{\varepsilon}\right)-C_{F}=\gamma u_{\varepsilon}^{\gamma-1}-C_{F} \geq 0
$$

We are now ready to establish the first Hausdorff type estimate for the level surface $\left\{u_{\varepsilon} \sim \varepsilon^{\alpha}\right\}$.

Lemma 7.3. Given a subdomain $\Omega^{\prime} \Subset \Omega$, there exists a constant $C$ depending on $\Omega^{\prime}$ and universal parameters such that, for $X_{0} \in \Omega^{\prime} \cap\left\{u_{\varepsilon}>\varepsilon^{\alpha}\right\}$, with $u_{\varepsilon}\left(X_{0}\right)=C_{1} \varepsilon^{\alpha}$, with $C_{1}>1$ and $\varepsilon \ll \rho$ for $\rho$ universally small, there holds

$$
\int_{\left\{C_{1} \varepsilon^{\alpha}<u_{\varepsilon}<\mu^{\alpha}\right\} \cap B_{\rho}\left(X_{0}\right)} u_{\varepsilon}^{-\gamma}\left|\nabla u_{\varepsilon}\right|^{2} d X \leq C \mu \rho^{N-1},
$$

for a.e. $0<\rho \ll 1$.

Proof. The proof starts off by verifying that for $\varepsilon$ and $\rho$ universally small, the following differential inequality holds:

$$
\sum_{i j} f_{i j} D_{i j}\left(u_{\varepsilon}^{\frac{1}{\alpha}}\right) \geq 0 \quad \text { in }\left\{u_{\varepsilon}>\varepsilon^{\alpha}\right\} \cap B_{\rho}\left(X_{0}\right) .
$$

where $\mathscr{F}=\left(f_{i j}\right)_{i j}$, as in Definition (7.1). To show such an estimate, we argue as follows: fix a non-singular linear operator $A: \mathbb{R}^{N} \rightarrow \mathbb{R}^{N}$. We compute

$$
\begin{aligned}
\sum_{i j} f_{i j} D_{i j}\left(u_{\varepsilon}^{\frac{1}{\alpha}}\right) & =\frac{1}{\alpha} u_{\varepsilon}^{\frac{1}{\alpha}-1} \operatorname{Tr}\left(A^{-1} \mathscr{F}\left(A^{-1}\right)^{T} D^{2}\left(u_{\varepsilon} \circ A\right)\right) \circ A^{-1} \\
& +\frac{1}{\alpha}\left(\frac{1}{\alpha}-1\right) u_{\varepsilon}^{\frac{1}{\alpha}-2} \operatorname{Tr}\left(A^{-1} \mathscr{F}\left(A^{-1}\right)^{T} \nabla\left(u_{\varepsilon} \circ A\right) \otimes \nabla\left(u_{\varepsilon} \circ A\right)\right) \circ A^{-1} .
\end{aligned}
$$


In addition, $u_{\varepsilon} \circ A$ solves the following uniform elliptic fully nonlinear equation

$$
F_{A}\left(D^{2} v\right)=\gamma v^{\gamma-1}
$$

where the operator $F_{A}$ is given by

$$
F_{A}(\mathscr{M}):=F\left(\left(A^{-1}\right)^{T} \mathscr{M} A^{-1}\right) .
$$

Easily one verifies that $F_{A}$ is in fact uniformly elliptic, with the same ellipticity constants as $F$. Thus, by optimal gradient bounds, we obtain

$$
\left|\nabla\left(u_{\varepsilon} \circ A\right)\right|^{2} \leq C\left(u_{\varepsilon} \circ A\right)^{\gamma} .
$$

and so,

$$
\frac{1}{\alpha}\left(\frac{1}{\alpha}-1\right)\left|\nabla\left(u_{\varepsilon} \circ A\right)\right|^{2} \geq \frac{C}{\alpha}\left(\frac{1}{\alpha}-1\right)\left(u_{\varepsilon} \circ A\right)^{\gamma} .
$$

From the structural assumption (AC),

$$
\begin{aligned}
\operatorname{Tr}\left(\left(A^{-1}\right)^{T} \mathscr{F} A^{-1} D^{2}\left(u_{\varepsilon} \circ A\right)\right) & \geq F\left(\left(A^{-1}\right)^{T} D^{2}\left(u_{\varepsilon} \circ A\right) A^{-1}\right)-C_{F} \\
& \geq F\left(\left(D^{2} u_{\varepsilon}\right) \circ A\right)-C_{F} \\
& \geq \gamma\left(u_{\varepsilon} \circ A\right)^{\gamma-1}-C_{F} .
\end{aligned}
$$

Thus, if we select $A$ as to satisfies

$$
\mathscr{F}=\frac{1}{C} A A^{T}
$$

where $C>0$ is the constant of inequality (7.3), and combine 7.3, 7.4 and 7.2, we end up with

$$
\sum_{i j} f_{i j} D_{i j}\left(u_{\varepsilon}^{\frac{1}{\alpha}}\right) \geq \frac{1}{\alpha} u_{\varepsilon}^{\frac{1}{\alpha}-1}\left(\frac{\gamma}{2} u_{\varepsilon}^{\gamma-1}-C_{F}\right) .
$$

Finally from Remark 7.2, we deduce that for $\varepsilon$ and $\rho$ universally small, the differential inequality (7.1) indeed holds true.

We now continue with the proof of Lemma 7.3. Define the following cut off function,

$$
\Phi=\left\{\begin{array}{cll}
\varepsilon \sqrt[\alpha]{C_{1}} & \text { in } & \left\{u_{\varepsilon} \leq C_{1} \varepsilon^{\alpha}\right\} \\
u_{\varepsilon}^{\frac{1}{\alpha}} & \text { in } & \left\{C_{1} \varepsilon^{\alpha}<u_{\varepsilon} \leq \mu^{\alpha}\right\} \\
\mu & \text { in } & \left\{u_{\varepsilon}>\mu^{\alpha}\right\}
\end{array}\right.
$$

Clearly we have

$$
\int_{\left\{C_{1} \varepsilon^{\alpha}<u_{\varepsilon} \leq \mu^{\alpha}\right\} \cap B_{\rho}\left(X_{0}\right)} f_{i j}\left(u_{\varepsilon}^{\frac{1}{\alpha}}\right)_{i} \cdot\left(u_{\varepsilon}^{\frac{1}{\alpha}}\right)_{j} d X=\int_{B_{\rho}\left(X_{0}\right)} f_{i j} \Phi_{i}\left(u_{\varepsilon}^{\frac{1}{\alpha}}\right)_{j} d X .
$$


Standard integrations by parts yield

$$
\begin{aligned}
\int_{B_{\rho}\left(X_{0}\right)} f_{i j} \Phi_{i}\left(u_{\varepsilon}^{\frac{1}{\alpha}}\right)_{j} d X & =-\int_{B_{\rho}\left(X_{0}\right)} \Phi \cdot f_{i j}\left(u_{\varepsilon}^{\frac{1}{\alpha}}\right)_{i j} d X \\
& +\frac{1}{\rho} \int_{\partial B_{\rho}\left(X_{0}\right)} f_{i j} \phi\left(u_{\varepsilon}^{\frac{1}{\alpha}}\right)_{i} \cdot\left(x^{j}-x_{0}^{j}\right) d \mathscr{H}^{N-1} .
\end{aligned}
$$

Therefore, from the differential inequality established in (7.1), we conclude

$$
\int_{\left\{C_{1} \varepsilon^{\alpha}<u_{\varepsilon} \leq \mu^{\alpha}\right\} \cap B_{\rho}\left(X_{0}\right)} f_{i j}\left(u_{\varepsilon}^{\frac{1}{\alpha}}\right)_{i}\left(u_{\varepsilon}^{\frac{1}{\alpha}}\right)_{j} d X \leq \frac{1}{\rho} \int_{\partial B_{\rho}\left(X_{0}\right)} \Phi \cdot f_{i j}\left(u_{\varepsilon}^{\frac{1}{\alpha}}\right)_{i} \cdot\left(x^{j}-x_{0}^{j}\right) d \mathscr{H}^{N-1} .
$$

Passing the derivatives through, we can further write the above estimate as

$$
\int_{\left\{C_{1} \varepsilon^{\alpha}<u_{\varepsilon} \leq \mu^{\alpha}\right\} \cap B_{\rho}\left(X_{0}\right)} f_{i j} u_{\varepsilon}^{-\gamma} D_{i} u_{\varepsilon} D_{j} u_{\varepsilon} d x \leq \frac{\alpha}{\rho} \int_{\partial B_{\rho}\left(X_{0}\right)} \Phi \cdot f_{i j} u_{\varepsilon}^{-\frac{\gamma}{2}} D_{i} u_{\varepsilon} \cdot\left(x^{j}-x_{0}^{j}\right) d \mathscr{H}^{N-1} .
$$

Finally, from uniform ellipticity and optimal regularity of $u_{\varepsilon}$, we derive

$$
\int_{\left\{C_{1} \varepsilon^{\alpha}<u_{\varepsilon} \leq \mu^{\alpha}\right\} \cap B_{\rho}\left(X_{0}\right)} u^{-\gamma|\nabla u|^{2} d X \leq C \mu \rho^{N-1},}
$$

as desired.

For the next result, let is recall the following classical notation: given a set $G \subset \mathbb{R}^{N}$, we will denote

$$
\mathscr{N}_{\delta}(G):=\left\{X \in \mathbb{R}^{N} \mid \operatorname{dist}(X, G)<\delta\right\} .
$$

In the sequel we show the main step towards uniform bounds of the $\mathscr{H}^{N-1}$-Hausdorff measure of the level-surfaces $\left\{u_{\varepsilon}>\varepsilon^{\alpha}\right\}$.

Lemma 7.4. Fixed $\Omega^{\prime} \Subset \Omega$, there exists a constant $C^{\star}$ that depends only on $\Omega^{\prime}$ and universal parameters such that if,

$$
C^{\star} \mu \leq 2 \rho \leq \frac{\operatorname{dist}\left(\Omega^{\prime}, \partial \Omega\right)}{10}
$$

then, for $\mu, \varepsilon>0$ universally small and $\mu \ll \rho$, for $\rho$ also universally small, we have

$$
\mathcal{L}^{N}\left(\left\{C_{1} \varepsilon^{\alpha}<u_{\varepsilon}<\mu^{\alpha}\right\} \cap B_{\rho}\left(X_{0}\right)\right) \leq \bar{C} \mu \rho^{N-1},
$$

where again $\bar{C}=\bar{C}\left(\Omega^{\prime}\right)$ depends only on $\Omega^{\prime}$ and universal constants and $X_{0} \in \Omega^{\prime} \cap \partial\left\{C_{1} \varepsilon^{\alpha}<u_{\varepsilon}<\right.$ $\left.\mu^{\alpha}\right\}$, with $d_{\varepsilon}\left(X_{0}\right) \leq \frac{\operatorname{dist}\left(\Omega^{\prime}, \partial \Omega\right)}{10}$ and $C_{1}>1$. 
Proof. Let $\left\{B_{j}\right\}$ be a finite family of balls covering $\partial\left\{C_{1} \varepsilon^{\alpha}<u_{\varepsilon}\right\} \cap B_{\rho}\left(X_{0}\right)$, with radius constant equal to $C^{\star} \mu$ and center $X_{j} \in \partial\left\{C_{1} \varepsilon^{\alpha}<u_{\varepsilon}\right\} \cap B_{\rho}\left(X_{0}\right)$, where $C^{\star}$ will be chosen a posteriori. By Heine-Borel Lemma, there exists a universal constant $m$ such that

$$
\sum_{j} \chi_{B_{j}} \leq m
$$

We can assure that

$$
\bigcup_{j} B_{j} \subset\left[\mathscr{N}_{\frac{d}{8}}\left(\Omega^{\prime}\right) \cap B_{4 \rho}\left(X_{0}\right)\right] .
$$

where $d:=\operatorname{dist}\left(\Omega^{\prime}, \partial \Omega\right)$. As in the proof of Lemma7.3, we consider

$$
\Phi=\left\{\begin{array}{cll}
\varepsilon \sqrt[\alpha]{C_{1}} & \text { in } & \left\{u_{\varepsilon} \leq C_{1} \varepsilon^{\alpha}\right\} ; \\
u_{\varepsilon}^{\frac{1}{\alpha}} & \text { in }\left\{C_{1} \varepsilon^{\alpha}<u_{\varepsilon} \leq \mu^{\alpha}\right\} \\
\mu & \text { in }\left\{u_{\varepsilon}>\mu^{\alpha}\right\} .
\end{array}\right.
$$

We now claim that is possible to find, for each $j$, balls $B_{j}^{1}$ and $B_{j}^{2}$ both contained in $B_{j}$, satisfying:

(1) the radius of $B_{j}^{1}$ and $B_{j}^{2}$ are in order $\mu$ (up to universal contraction)

(2) $\Phi \geq \sqrt[\alpha]{\frac{3}{4}} \mu$ in $B_{j}^{1}$ and $\Phi \leq \sqrt[\alpha]{\frac{2}{3}} \mu$ in $B_{j}^{2}$.

To show the above claim, we argue as follows: take $X_{1} \in \frac{1}{4} \overline{B_{j}}$, such that

$$
u_{\varepsilon}\left(X_{1}\right)=\sup _{\frac{1}{4} B_{j}} u_{\varepsilon}
$$

By strong nondegeneracy,

$$
u_{\varepsilon}\left(X_{1}\right) \geq c_{0}\left(\frac{C^{\star} \mu}{4}\right)^{\alpha} \geq C_{2} \mu^{\alpha},
$$

if $C^{\star} \gg 1$ is chosen universally large enough, where $C_{2}>0$ is a constant obteined from Theorem 4.2. This last theorem, given $X \in B_{j}$, we obtain

$$
u_{\varepsilon}(X) \geq \frac{1}{C_{2}} u\left(X_{1}\right)-\left|X-X_{1}\right|^{\alpha} \geq \mu^{\alpha}-\left|X-X_{1}\right|^{\alpha} .
$$

Taking

we obtain

$$
\left|X-X_{1}\right|<\sqrt[\alpha]{\frac{1}{4}} \mu
$$

$$
\Phi^{\alpha}(X)=u_{\varepsilon}(X) \geq \frac{3}{4} \mu^{\alpha} \quad \text { in } B_{j}^{1}:=B_{r_{j}^{1}}\left(X_{1}\right)
$$

where $r_{j}^{1}:=\sqrt[\alpha]{\frac{1}{4}}$ is a universal constant. To finish up the proof of this first statement, we just choose $C^{\star}$ large enough as to

$$
\tilde{C}_{1} \ll C^{\star} \quad \Longrightarrow \quad B_{j}^{1} \subset B_{j}
$$


Notice again that such a selection is universal. Similarly, for $B_{j}^{2}:=B_{r_{j}^{2}}\left(X_{j}\right)$ where $r_{j}^{2}:=\tilde{C}_{2} \mu \ll$ $C^{\star} \mu$, we have

$$
\Phi(X)^{\alpha}=u_{\varepsilon} \leq \frac{2}{3} \mu^{\alpha}
$$

From property (2) proven above, assures the existence of a universal constant $\kappa>0$ such that, for each $j \in \mathbb{N}$

$$
\left|\Phi-m_{j}\right|>\kappa \mu
$$

in at least one the two balls $B_{j}^{1}, B_{j}^{2} \subset B_{j}$, where

$$
m_{j}:=f_{B_{j}} \Phi(X) d X
$$

Thus, by classical Poincaré inequality in balls, we derive

$$
\kappa^{2} \mu^{2} \leq \frac{1}{\left|B_{j}\right|} \int_{B_{j}}\left|\Phi-m_{j}\right|^{2} d X \leq C_{3} \mu^{2} \frac{1}{\left|B_{j}\right|} \int_{B_{j}}|\nabla \Phi|^{2} d X,
$$

which in turn gives

$$
\int_{\left\{C_{1} \varepsilon^{\alpha}<u_{\varepsilon}<\mu^{\alpha}\right\} \cap B_{j}} u_{\varepsilon}^{-\gamma}\left|\nabla u_{\varepsilon}\right|^{2} d x \geq C_{4}\left|B_{j}\right|
$$

In addition, by nondegeneracy, for all $Y \in\left\{C_{1} \varepsilon^{\alpha}<u_{\varepsilon}<\mu^{\alpha}\right\} \cap B_{\rho}\left(X_{0}\right)$, we have

$$
C_{5} d_{\varepsilon}(Y)^{\alpha} \leq u_{\varepsilon}(Y) \leq \mu^{\alpha}
$$

Hence

$$
\left\{C_{1} \varepsilon^{\alpha}<u_{\varepsilon}<\mu^{\alpha}\right\} \cap B_{\rho}\left(X_{0}\right) \subset \mathscr{N}_{\frac{1}{C_{6}} \mu}\left(\partial\left\{C_{1} \varepsilon^{\alpha}<u_{\varepsilon}\right\} \cap B_{2 \rho}\left(X_{0}\right)\right),
$$

for $C_{6}=\sqrt[\alpha]{C_{5}}$. Thus, for $\mu \ll \rho$, and $C^{\star} \gg 1$, both universal, we reach

$$
\left\{C_{1} \varepsilon^{\alpha}<u_{\varepsilon}<\mu^{\alpha}\right\} \cap B_{\rho}\left(X_{0}\right) \subset \bigcup 2 B_{j} \subset B_{4 \rho}\left(X_{0}\right) .
$$

Finally, applying Lemma (7.3) and taking into account the inclusion above, we estimate

$$
\begin{aligned}
C_{7} \mu \rho^{N-1} & \geq \int_{B_{4 \rho}\left(X_{0}\right) \cap\left\{C_{1} \varepsilon^{\alpha}<u_{\varepsilon}<\mu^{\alpha}\right\}} u_{\varepsilon}^{-\gamma}\left|\nabla u_{\varepsilon}\right|^{2} d x \\
& \geq \frac{1}{m} \sum \int_{2 B_{j} \cap\left\{C_{1} \varepsilon^{\alpha}<u_{\varepsilon}<\mu^{\alpha}\right\}} u_{\varepsilon}^{-\gamma}\left|\nabla u_{\varepsilon}\right|^{2} d x \\
& \geq \frac{C_{4}}{m} \sum\left|B_{j}\right| \\
& \geq \frac{C_{4}}{m}\left|B_{\rho}\left(X_{0}\right) \cup\left\{C_{1} \varepsilon^{\alpha}<u_{\varepsilon}<\mu^{\alpha}\right\}\right|,
\end{aligned}
$$

where $C_{7}$ and $C_{4}$ are universal constants, which completes the proof of Lemma. 
In the sequel, we recall the definition of $\delta$-density.

Definition 7.5. Given an open subset $G$ of $\mathbb{R}^{N}$, we say that $G$ has the $\delta$-density property in $\Omega$ for $0<\delta<1$, if there exists $\tau>0$ such that

$$
\frac{\mathcal{L}^{N}\left(B_{\delta}(X) \cap G\right)}{\mathcal{L}^{N}\left(B_{\delta}(X)\right)} \geq \tau
$$

for all $X \in \partial G \cap \Omega$. If the property above is valid for any $0<\delta<1$, we say that $G$ has uniform density in $\Omega$ along $\partial G$.

Here we state a, by now, classical result from measure theory.

Lemma 7.6. Given an open set $A \Subset \Omega$, there holds:

a) If there exists $\delta$ such that A has the $\delta$-density property, then there exists a constant $C=C(\tau, N)$, where:

$$
\left|\mathscr{N}_{\delta}(\partial A) \cap B_{\rho}(X)\right| \leq \frac{1}{2^{N} \tau}\left|\mathscr{N}_{\delta}(\partial A) \cap B_{\rho}(X) \cap A\right|+C \delta \rho^{N-1}
$$

with $X \in \partial A \cap \Omega$ and $\delta \ll \rho$.

b) If $A$ has uniform density in $\Omega$ along $A$, then $|\partial A \cap \Omega|=0$.

We are ready to state and prove the main result on this section.

Theorem 7.7. Given $\Omega^{\prime} \Subset \Omega$ there exists a universal constant $C=C\left(\Omega^{\prime}\right)>0$, such that

$$
\mathcal{L}^{N}\left(\mathscr{N}_{\mu}\left(\left\{C_{1} \varepsilon^{\alpha}<u_{\varepsilon}\right\}\right) \cap B_{\rho}\left(X_{0}\right)\right) \leq C \mu \rho^{N-1},
$$

whenever, $C_{1}>1, X_{0} \in \Omega^{\prime} \cap \partial\left\{C_{1} \varepsilon^{\alpha}<u_{\varepsilon}\right\}, d_{\varepsilon}\left(X_{0}\right)<\frac{1}{10} \operatorname{dist}\left(\Omega^{\prime}, \partial \Omega\right), \mu \ll \rho$ with $\rho$ universally small and $C_{1} \varepsilon^{\alpha}<\mu^{\alpha}$. In particular,

$$
\mathscr{H}^{N-1}\left(\partial\left\{C_{1} \varepsilon^{\alpha}<u_{\varepsilon}\right\} \cap B_{\rho}\left(X_{0}\right)\right) \leq C \rho^{N-1} .
$$

Proof. Take $\mu=\delta$, is as in the statement of Corollary (5.6). We have,

$$
\frac{\mathcal{L}^{N}\left(B_{\delta}(X) \cap\left\{u_{\varepsilon}>C_{1} \varepsilon^{\alpha}\right\}\right)}{\mathcal{L}^{N}\left(B_{\delta}(X)\right)} \geq C_{2},
$$

for $X \in \partial\left\{u_{\varepsilon}>C_{1} \varepsilon^{\alpha}\right\}$. We conclude that, $\partial\left\{u_{\varepsilon}>C_{1} \varepsilon^{\alpha}\right\}$ has the $\delta$-density property, and by Lemma 7.6, for a universal constant $M>0$, there holds

$$
\begin{aligned}
\mathcal{L}^{N}\left(\mathscr{N}_{\delta}\left(\partial\left\{u_{\varepsilon}>C_{1} \varepsilon^{\alpha}\right\}\right) \cap B_{\rho}\left(X_{0}\right)\right) & \leq \frac{1}{2^{N} C_{2}} \mathcal{L}^{N}\left(\mathscr{N}_{\delta}\left(\partial\left\{u_{\varepsilon}>C_{1} \varepsilon^{\alpha}\right\}\right) \cap B_{\rho}\left(X_{0}\right) \cap\left\{u_{\varepsilon}>C_{1} \varepsilon^{\alpha}\right\}\right) \\
& +M \delta \rho^{N-1} .
\end{aligned}
$$

From Theorem 4.2 given $Y \in \mathscr{N}_{\delta}\left(\partial\left\{u_{\varepsilon}>C_{1} \varepsilon^{\alpha}\right\}\right) \cap B_{\rho}\left(X_{0}\right) \cap\left\{u_{\varepsilon}>C_{1} \varepsilon^{\alpha}\right\}$ and $Z \in \partial\left\{u_{\varepsilon}>C_{1} \varepsilon^{\alpha}\right\}$, we can estimate

$$
\begin{aligned}
u(Y) & \leq C_{3}\left(|Z-Y|^{\alpha}+u(Z)\right) \\
& \leq C_{3}\left(\delta^{\alpha}+\mu^{\alpha}\right) \\
& \leq D \mu^{\alpha}
\end{aligned}
$$


where the last inequality, follows from $C_{1} \varepsilon^{\alpha}<\mu$ and $\delta=C \mu$. We have verified there exists $D>0$ universal, such that

$$
\mathscr{N}_{\delta}\left(\partial\left\{u_{\varepsilon}>C_{1} \varepsilon^{\alpha}\right\}\right) \cap B_{\rho}\left(X_{0}\right) \cap\left\{u_{\varepsilon}>C_{1} \varepsilon^{\alpha}\right\} \subset\left\{C_{1} \varepsilon^{\alpha}<u_{\varepsilon}<D \mu^{\alpha}\right\} \cap B_{\rho}\left(X_{0}\right) .
$$

Finally, from Lemma (7.4), we conclude

$$
\mathcal{L}^{N}\left(\left\{C_{1} \varepsilon^{\alpha}<u_{\varepsilon}<D \mu^{\alpha}\right\} \cap B_{\rho}\left(X_{0}\right)\right) \leq C_{4} \mu \rho^{N-1},
$$

thus, combing (7.8), (7.9) and (7.10) we reach,

$$
\mathcal{L}^{N}\left(\mathscr{N}_{\mu}\left(\left\{u_{\varepsilon}>C_{1} \varepsilon^{\alpha}\right\}\right) \cap B_{\rho}\left(X_{0}\right)\right) \leq C_{4} \mu \rho^{N-1} .
$$

To conclude the proof of the $\mathscr{H}^{N-1}$ Hausdorff measure estimate, let $\left\{B_{j}\right\}$ be a covering of $\partial\left\{C_{1} \varepsilon^{\alpha}<u_{\varepsilon}\right\} \cap B_{\rho}\left(X_{0}\right)$, where each ball be centered in $\partial\left\{C_{1} \varepsilon^{\alpha}<u_{\varepsilon}\right\} \cap B_{\rho}\left(X_{0}\right)$ with radius $\mu$. We can write

$$
\bigcup B_{j} \subset \mathscr{N}_{\mu}\left(\left\{C_{1} \varepsilon^{\alpha}<u_{\varepsilon}\right\}\right) \cap B_{\rho+\mu}\left(X_{0}\right) .
$$

Thus there exist dimensional constants $C_{5}, C_{6}>0$, such that

$$
\begin{aligned}
\mathscr{H}_{\mu}^{N-1}\left(\partial\left\{C_{1} \varepsilon^{\alpha}<u_{\varepsilon}\right\} \cap B_{\rho}\left(X_{0}\right)\right) & \leq C_{5} \sum \operatorname{Area}\left(\partial B_{j}\right) \\
& =\frac{C_{5}}{\mu} \sum \mathcal{L}^{N}\left(B_{j}\right) \\
& \leq \frac{C_{6}}{\mu} \mathcal{L}^{N}\left(\mathscr{N}_{\mu}\left(\left\{C_{1} \varepsilon^{\alpha}<u_{\varepsilon}\right\}\right) \cap B_{\rho+\mu}\left(X_{0}\right)\right) \\
& \leq C_{6} C_{4}(\rho+\mu)^{N-1}=C_{6} C_{4} \rho^{N-1}+\mathrm{o}(1) .
\end{aligned}
$$

Letting $\mu \rightarrow 0$, we finish the proof of the Theorem.

\section{Limiting free boundary problem}

In this Section, we address the fully nonlinear free boundary problem obtained by letting $\varepsilon \rightarrow 0$. The ultimate goal is to find a solution to the free boundary problem (1.2) that enjoys all the desired analytic and geometric properties.

Our analysis starts off by the compactness of minimal solutions to Equation $\left(E_{\varepsilon}\right)$. In fact, Proposition (4.1) implies that $\left\{u_{\varepsilon}\right\}_{\varepsilon>0}$ is a compact sequence and up to a subsequence,

$$
\lim _{\varepsilon \rightarrow 0} u_{\varepsilon}=: u_{0} .
$$

This Section is devoted to the study of the limiting function $u_{0}$ and the free boundary problem it solves. 
For the readers convenience, let us hereafter set up the following notations that we will use throughout this Section:

$$
\begin{aligned}
\left\{u_{0}>0\right\} & :=\left\{x \in \Omega \mid u_{0}(x)>0\right\}, \\
\mathfrak{F}\left(u_{0}\right) & :=\partial\left\{u_{0}>0\right\} \cap \Omega, \\
d_{0}(X) & :=\operatorname{dist}\left(X, \mathfrak{F}\left(u_{0}\right)\right) .
\end{aligned}
$$

Next Theorem recovers the fully nonlinear equation satisfies by $u_{0}$ within its positive set as well as its precise growth behavior near the free boundary, $\mathfrak{F}\left(u_{0}\right)$.

Theorem 8.1. The limiting function $u_{0}$ defined in (8.1) is a viscosity solution to

$$
F\left(D^{2} u\right)=\gamma u^{\gamma-1} \quad \text { in }\{u>0\}
$$

Moreover, for a fixed $\Omega^{\prime} \Subset \Omega$, there exists a constant $C=C\left(\Omega^{\prime}\right)$ that depends on $\Omega^{\prime}$ and universal constants such that for any $X \in \Omega^{\prime} \cap\left\{u_{0}>0\right\}$, there holds

$$
C d_{0}(X)^{\alpha} \leq u_{0}(X) \leq C^{-1} d_{0}(X)^{\alpha}
$$

whenever $d_{0}(X) \leq \frac{\operatorname{dist}\left(\Omega^{\prime}, \partial \Omega\right)}{4}$. In particular, $u_{0}$ is a solution to the free boundary problem (1.2).

Proof. Let us fix a point $X_{0} \in\left\{u_{0}>0\right\}$ and let $u_{0}\left(X_{0}\right):=\sigma>0$. By continuity $u_{0} \geq \frac{1}{2} \sigma$ in $B_{\rho}\left(X_{0}\right)$ for same $\rho>0$. Since $u_{\varepsilon} \rightarrow u_{0}$ uniformly over compact sets, for $\varepsilon \ll 1$ we have

$$
u_{\varepsilon} \geq \frac{1}{8} \sigma>\left(1+\sigma_{0}\right) \varepsilon^{\alpha}
$$

That is, $u_{\varepsilon}$ satisfies

$$
F\left(D^{2} u_{\varepsilon}\right)=\gamma u_{\varepsilon}^{\gamma-1} \text { in } B_{\frac{1}{2} \rho}\left(X_{0}\right) .
$$

By the stability of viscosity solutions under uniform limits, we conclude $u_{0}$ is indeed a viscosity solution to Equation (8.2).

Let us now turn our attention to the growth rate controls. For that, fix $X_{0} \in \Omega^{\prime} \cap\left\{u_{0}>0\right\}$, with $d_{0}\left(X_{0}\right) \leq \frac{1}{4} \operatorname{dist}\left(\Omega^{\prime}, \partial \Omega\right)$ and label $u_{0}\left(X_{0}\right)=s>0$. For $\varepsilon \ll 1$ we have

$$
u_{\varepsilon}\left(X_{0}\right) \geq \frac{s}{2}>\varepsilon^{\alpha}
$$

Thus, according to Corollary 5.4, we obtain

$$
u_{\varepsilon}\left(X_{0}\right) \geq C d_{\varepsilon}\left(X_{0}\right)^{\alpha}
$$

Let $Y_{\varepsilon} \in \partial\left\{u_{\varepsilon}>\varepsilon^{\alpha}\right\}$ be such that $d_{\varepsilon}\left(X_{0}\right)=\left|X_{0}-Y_{\varepsilon}\right|$. By uniform convergence, it clearly follows that $Y_{\varepsilon} \rightarrow Y_{0}$ and $u_{0}\left(Y_{0}\right)=0$. In conclusion,

$$
u_{0}\left(X_{0}\right) \geq C\left|X_{0}-Y_{0}\right|^{\alpha} \geq C d_{0}\left(X_{0}\right)^{\alpha} .
$$

The upper estimate is obtained similarly. 
Strong nondegeneracy property established for the approximating solutions $u_{\varepsilon}$ also passes to the limiting configuration.

Theorem 8.2. Given $\Omega^{\prime} \Subset \Omega$, there exist universal constants $C, \rho_{0}>0$, depending only on $\Omega^{\prime}$ and universal constants, such that for any $X \in \Omega^{\prime} \cap \overline{\left\{u_{0}>0\right\}}, \rho \leq \rho_{0}$ and $d_{0}(X)<\frac{\operatorname{dist}\left(X, \partial \Omega^{\prime}\right)}{2}$, there holds

$$
C^{-1} \rho^{\alpha} \leq \sup _{B_{\rho}(X)} u_{0} \leq C\left(\rho^{\alpha}+u_{0}(X)\right)
$$

The proof of Theorem 8.2 is very similar to the one presented for Theorem 8.1 and therefore,we shall omit the details. Next we show the approximating configurations $\left\{u_{\varepsilon}\right\}$ converge to the liming one, $\left\{u_{0}>0\right\}$ in the Hausdorff metric.

Theorem 8.3. Given $\delta>0$ and $\varepsilon \ll 1$, we following inclusions hold:

$$
\left\{u_{0}>0\right\} \cap \Omega^{\prime} \subset \mathscr{N}_{\delta}\left(\left\{u_{\varepsilon}>C_{1} \varepsilon^{\alpha}\right\}\right) \cap \Omega^{\prime} \quad \text { and } \quad\left\{u_{\varepsilon}>C_{1} \varepsilon^{\alpha}\right\} \cap \Omega^{\prime} \subset \mathscr{N}_{\delta}\left(\left\{u_{0}>0\right\}\right) \cap \Omega^{\prime} .
$$

Proof. We will show only the last inclusion, as the first follows similarly. Suppose, for the purpose of contradiction, that such inclusion is false. There would exist, therefore, $\delta_{0}>0$ and a sequence of points $\left\{X_{\varepsilon}\right\}$, satisfying

a) $X_{\varepsilon} \in \Omega^{\prime} \cap\left\{u_{\varepsilon}>C_{1} \varepsilon^{\alpha}\right\}$;

b) $\operatorname{dist}\left(X_{\varepsilon},\left\{u_{0}>0\right\}\right)>\delta_{0}$;

c) $X_{\varepsilon} \rightarrow X_{0}$, and $\operatorname{dist}\left(X_{0},\left\{u_{0}>0\right\}\right)>\delta_{0}$.

From property c) $u_{0}\left(X_{0}\right)=0$. However, by strong non-degeneracy, Theorem (8.2), for each $\varepsilon$, we can find $Z_{\varepsilon} \in \overline{B_{\frac{1}{2}} \delta_{0}\left(X_{\varepsilon}\right)}$, such that

$$
u_{\varepsilon}\left(Z_{\varepsilon}\right)=\sup _{B_{\frac{1}{2} \delta_{0}}\left(X_{\varepsilon}\right)} u_{\varepsilon} \geq C \delta_{0}^{\alpha}
$$

As $\varepsilon \rightarrow 0$, up to a subsequence, $Z_{\varepsilon} \rightarrow Z_{0}$. However, from (8.3) and $u_{0}\left(Z_{0}\right)>0$ and by property c) above $Z_{0} \in\left\{u_{0}=0\right\}$, which is a contradiction.

It also follows as in Corollary 5.6 that the set $\left\{u_{0}>0\right\}$ has uniform positive density along the free boundary $\mathfrak{F}\left(u_{0}\right)$.

Theorem 8.4. Given $\Omega^{\prime} \Subset \Omega$ there exists a constant $0<c \leq 1$, depending on $\Omega^{\prime}$ and universal parameters, such that

$$
\frac{\mathcal{L}^{N}\left(B_{\delta}(X) \cap\left\{u_{0}>0\right\}\right)}{\mathcal{L}^{N}\left(B_{\delta}(X)\right)} \geq c
$$

for all $X \in \mathfrak{F}\left(u_{0}\right) \cap \Omega^{\prime}$. 
The proof of Theorem 8.4 is similar to the one presented for Corollary 5.6 and therefore we omit the details.

As a consequence of the analysis carried out in Section 6, we will show that a clean Harnack inequality is valid near the free boundary $\mathfrak{F}\left(u_{0}\right)$. As mentioned in that Section, such a result is quite surprising a first view, as the nonlinear source of the equation is of order $\sim u^{\gamma-1}$ and thus it blows up near the boundary of the quenching region.

Theorem 8.5 (Harnack Inequality for tangential balls). Let $X_{0} \in\left\{u_{0}>0\right\}$ and $d:=d_{0}\left(X_{0}\right)$. Then, there exist a universal constant $C>0$ such that

$$
\sup _{B_{\frac{d}{2}}\left(X_{0}\right)} u_{0} \leq C \inf _{B_{\frac{d}{2}}\left(X_{0}\right)} u_{0}
$$

Proof. Let $\xi_{0}, \xi_{1} \in \overline{B_{\frac{d}{2}}\left(X_{0}\right)}$, be such that

$$
\inf _{B_{\frac{d}{2}}\left(X_{0}\right)} u_{0}=u_{0}\left(\xi_{0}\right) \quad \text { and } \quad \sup _{B_{\frac{d}{2}}\left(X_{0}\right)} u_{0}=u_{0}\left(\xi_{1}\right)
$$

Since $d_{0}\left(\xi_{0}\right) \geq \frac{d}{2}$, by Theorem 8.2 , there holds

$$
u_{0}\left(\xi_{0}\right) \geq C_{1} d^{\alpha}
$$

On the other hand, from Theorem 8.2 , we have

$$
u_{0}\left(\xi_{1}\right) \leq C_{2}\left(\frac{d^{\alpha}}{2}+u_{0}\left(X_{0}\right)\right)
$$

and for $Y \in \partial\left\{u_{0}>0\right\}$ where $d=\left|Y-X_{0}\right|$,

$$
u_{0}\left(X_{0}\right) \leq \sup _{B_{d}(Y)} u_{0} \leq C_{2} d^{\alpha}
$$

Thus,

$$
\sup _{B_{\frac{d}{2}}\left(X_{0}\right)} u_{0} \leq C_{2} \inf _{B_{\frac{d}{2}}\left(X_{0}\right)} u_{0}
$$

for a constant $C_{2}>0$ that does not depend of $u_{0}$.

As in the proof of Corollary 6.1, we can establish a lower bound for solid integrals for $u_{0}$ : for all $X_{0} \in \partial\left\{u_{0}>0\right\} \cap \Omega^{\prime}$

$$
C_{1} \rho^{\alpha} \leq f_{B_{\rho}\left(X_{0}\right)} u_{0} d x
$$

where $C_{1}=C_{1}\left(\Omega^{\prime}\right)>0$. Next we establish upper and lower control on spherical integrals of $u_{0}$. 
Theorem 8.6. Given $\Omega^{\prime} \Subset \Omega$, there exists a universal constant $C=C\left(\Omega^{\prime}\right)$, such that for all $X_{0} \in$ $\partial\left\{u_{0}>0\right\} \cap \Omega^{\prime}$,

$$
C^{-1} \rho^{\alpha} \leq f_{\partial B_{\rho}\left(X_{0}\right)} u_{0} d \mathscr{H}^{N-1} \leq C \rho^{\alpha} .
$$

Proof. The upper estimate follows directly from Corollary (8.2). We will show the lower bound by means of contradiction. Suppose the lower inequality is not valid. There would then exist $\rho_{m}>0$ and $X_{m} \in \partial\left\{u_{0}>0\right\}$, such that

$$
\frac{1}{\rho_{m}^{\alpha}} f_{\partial B_{\rho_{m}}\left(X_{m}\right)} u_{0} d \mathscr{H}^{N-1}=\mathrm{o}(1),
$$

as $m \rightarrow \infty$. Clearly, (8.6) implies

$$
\frac{1}{\rho_{m}^{\alpha}} f_{\partial B_{r \rho_{m}}\left(X_{m}\right)} u_{0} d \mathscr{H}^{N-1}=\mathrm{o}(1),
$$

for all $0<r \leq 1$. Define

$$
v_{m}(X):=\rho_{m}^{-\alpha} u_{0}\left(X_{m}+\rho_{m} X\right) .
$$

Up to a subsequence, $v_{m}$ converges uniformly over compact subsets of $\mathbb{R}^{N}$, to a function $v_{0}$. Furthermore,

$$
F_{m}\left(D^{2} v_{m}\right)=\gamma v_{m}^{\gamma-1} \text { in }\left\{v_{m}>0\right\},
$$

where $F_{m}(\mathscr{M}):=\rho_{m}^{\alpha} F\left(\rho_{m}^{-\alpha} \mathscr{M}\right)$. For any $0<r \leq 1$,

$$
f_{\partial B_{r \rho_{m}}\left(X_{m}\right)} u_{0}(Y) d \mathscr{H}^{N-1}=f_{\partial B_{r}(0)} u_{0}\left(X_{m}+\rho_{m} X\right) d \mathscr{H}^{N-1}=\rho_{m}^{\alpha} f_{\partial B_{r}(0)} v_{m}(X) d \mathscr{H}^{N-1} .
$$

Thus, by (8.7), letting $m \rightarrow \infty$, yields

$$
f_{\partial B_{r}(0)} v_{0} d \mathscr{H}^{N-1}=\rho_{m}^{-\alpha} f_{\partial B_{r \rho_{m}}\left(X_{m}\right)} u_{0} d \mathscr{H}^{N-1}=0, \quad \forall 0<r \leq 1 .
$$

Therefore, $v_{0} \equiv 0$ in $B_{1}$ which contradicts $(8.5)$ properly scaled to $v_{m}$.

\section{Geometric estimates of the free boundary}

In this final Section we obtain further fine geometric-measure properties of the free boundary $\mathfrak{F}\left(u_{0}\right)$. As in Section 7 , here we shall work under the addition structural assumption (AC). The first result we show concerns the local finiteness of the $\mathscr{H}^{N-1}$-Hausdorff measure of the free boundary $\mathfrak{F}\left(u_{0}\right)$.

Theorem 9.1. Given $\Omega^{\prime} \Subset \Omega$ there exists a constant $C=C\left(\Omega^{\prime}\right)>0$, depending on $\Omega^{\prime}$ and universal constants, such that

$$
\mathcal{L}^{N}\left(\mathscr{N}_{\mu}\left(\left\{u_{0}>0\right\}\right) \cap B_{\rho}\left(X_{0}\right)\right) \leq C \mu \rho^{N-1},
$$

wherenever, $X_{0} \in \Omega^{\prime} \cap \partial\left\{u_{0}>0\right\}, d_{0}\left(X_{0}\right)<\frac{1}{10} \operatorname{dist}\left(\Omega^{\prime}, \partial \Omega\right), \mu \ll \rho$ and $\rho$ is universally small. In particular,

$$
\mathscr{H}^{N-1}\left(B_{\rho}\left(X_{0}\right) \cap \mathfrak{F}\left(u_{0}\right)\right) \leq C \rho^{N-1} .
$$


Proof. From Theorem 7.7 and Theorem 8.3 , we have for $\varepsilon \ll 1$

$$
\begin{gathered}
\left|\mathscr{N}_{2 \mu}\left(\left\{u_{\varepsilon}>C_{1} \varepsilon^{\alpha}\right\}\right) \cap B_{\rho}\left(X_{0}\right)\right| \leq C \mu \rho^{N-1} \\
\text { and } \\
\left\{u_{0}>0\right\} \cap B_{\rho}\left(X_{0}\right) \subset \mathscr{N}_{\mu}\left(\left\{u_{\varepsilon}>C_{1} \varepsilon^{\alpha}\right\}\right) \cap B_{\rho}\left(X_{0}\right) .
\end{gathered}
$$

Easily we show

$$
\mathscr{N}_{\mu}\left(\left\{u_{0}>0\right\}\right) \cap B_{\rho}\left(X_{0}\right) \subset \mathscr{N}_{2 \mu}\left(\left\{u_{\varepsilon}>C_{1} \varepsilon^{\alpha}\right\}\right) \cap B_{\rho}\left(X_{0}\right),
$$

which give us the estimate desired.

A consequence of Theorem 9.1 is that the limiting region $\left\{u_{0}>0\right\}$ has locally finite perimeter. The key final result we will show here states that the reduced free boundary, $\partial_{\text {red }}\left\{u_{0}>0\right\}$ has total measure. More importantly, we prove that around points $Z$ of the reduced free boundary, there holds

$$
\mathscr{H}^{N-1}\left(B_{\rho}(Z) \cap \mathfrak{F}\left(u_{0}\right)\right) \sim \rho^{N-1} .
$$

In particular the free boundary has a theoretical measure outward unit vector for $\mathscr{H}^{N-1}$ almost all points in $\mathfrak{F}\left(u_{0}\right)$.

Theorem 9.2. Given $\Omega^{\prime} \Subset \Omega$, there exists a positive constant $C=C\left(\Omega^{\prime}\right)$, that depends only on $\Omega^{\prime}$ and universal constants, such that for any ball $B_{\rho}\left(X_{0}\right)$, with $\rho$ universally small, centered at a free boundary point $x_{0} \in \partial\left\{u_{0}>0\right\}$, there holds

$$
C^{-1} \rho^{N-1} \leq \mathscr{H}^{N-1}\left(\partial_{\text {red }}\left\{u_{0}>0\right\} \cap B_{\rho}\left(X_{0}\right)\right) \leq C \rho^{N-1} .
$$

In particular,

$$
\mathscr{H}^{N-1}\left(\partial\left\{u_{0}>0\right\} \backslash \partial_{\text {red }}\left\{u_{0}>0\right\}\right)=0 .
$$

Proof. The estimate from above follows from Theorem 9.1, It remains to verify the estimate by below. Fixed $X_{0}$, let us define the normalized function $v_{0}: B_{1} \rightarrow \mathbb{R}$ by

$$
v_{0}(X):=\frac{u_{0}\left(X_{0}-\rho X\right)}{\rho^{\alpha}} .
$$

Arguing as in the proof of Theorem (7.3), for $\rho$ universally small, we conclude,

$$
\mathrm{L}\left(v_{0}^{\frac{1}{\alpha}}\right):=\rho^{2-\alpha} \sum_{i j} f_{i j} D_{i j}\left(u_{0}^{\frac{1}{\alpha}}\right) \geq 0 \quad \text { in } \quad\left\{v_{0}>0\right\} \cap B_{1} .
$$

Our next step is to furnish an appropriate special barrier. Let $\psi$ be a nonnegative smooth function in $B_{1}$, with $\psi \equiv 1$ in $B_{1 / 5}$ and $\psi \equiv 0$ outside $B_{1 / 4}$. Let $\Phi$ be the solution to the following boundary value problem

$$
\left\{\begin{array}{ccccc}
\mathrm{L} \Phi & = & -\psi & \text { in } & B_{1} \\
\Phi & = & 0 & \text { on } & \partial B_{1}
\end{array}\right.
$$


From classical elliptic regularity theory, $\Phi$ is smooth and, in particular, for any $0<\alpha<1$,

$$
\|\Phi\|_{C^{\alpha}\left(B_{1 / 2}\right)} \leq C_{1}
$$

by a universal constant $C_{1}>0$. Also by maximum principle $\Phi>0$ in $B_{1}$ and by Hopf maximum principle,

$$
f_{i j} \partial_{i} \Phi v_{j} \geq C_{2}>0, \quad \text { along } \partial B_{1},
$$

where $v_{j}$ is the $j$-th coordinate of the outward normal vector to $\partial B_{1}(0)$. Applying generalized Gauss-Green formula, we derive

$$
\begin{aligned}
\int_{\left\{v_{0}>0\right\} \cap B_{1}}\left\{\Phi \mathrm{L}\left(v_{0}^{\frac{1}{\alpha}}\right)-v_{0}^{\frac{1}{\alpha}} \mathrm{L} \Phi\right\} d x & =\int_{\partial_{\operatorname{red}\{}\left\{v_{0}>0\right\} \cap B_{1}}\left\{\Phi f_{i j} \partial_{i}\left(v_{0}^{\frac{1}{\alpha}}\right)-v_{0}^{\frac{1}{\alpha}} f_{i j} \partial_{i} \Phi\right\} \eta_{j} d \mathscr{H}^{N-1} \\
& -\int_{\left\{v_{0}>0\right\} \cap \partial B_{1}} v_{0}^{\frac{1}{\alpha}} f_{i j} \partial_{i} \Phi v_{j} d \mathscr{H}^{N-1} .
\end{aligned}
$$

Since $\Phi \mathrm{L}\left(v_{0}^{\frac{1}{\alpha}}\right) \geq 0$, there holds

$$
\int_{\left\{v_{0}>0\right\} \cap B_{1}}\left\{\Phi \mathrm{L}\left(v_{0}^{\frac{1}{\alpha}}\right)-v_{0}^{\frac{1}{\alpha}} \mathrm{L} \Phi\right\} d x \geq \int_{B_{1}} \psi v_{0}^{\frac{1}{\alpha}} d x \geq \int_{B_{1 / 5}} v_{0}^{\frac{1}{\alpha}} d x .
$$

Also from uniform gradient bounds of $v_{0}$, ellipticity and (9.2) we estimate

$$
\left|\int_{\partial_{\text {red }}\left\{v_{0}>0\right\} \cap B_{1}} \Phi f_{i j} \partial_{i}\left(v_{0}^{\frac{1}{\alpha}}\right) \eta_{j} d \mathscr{H}^{N-1}\right| \leq C_{1} \mathscr{H}^{N-1}\left(\partial_{\text {red }}\left\{v_{0}>0\right\} \cap B_{1}\right) .
$$

In addition, clearly,

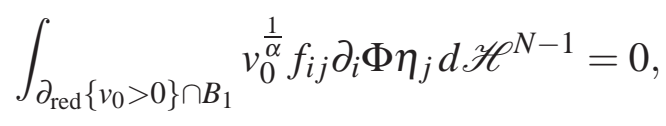

and by $(9.3)$,

$$
\int_{\left\{v_{0}>0\right\} \cap \partial B_{1}} v_{0}^{\frac{1}{\alpha}} f_{i j} \partial_{i} \Phi v_{j} d \mathscr{H}^{N-1} \geq 0 .
$$

Combining (9.4), (9.5), (9.6) and (9.7), we deduce

$$
\int_{B_{1 / 5}} v_{0}^{\frac{1}{\alpha}} d x \leq C_{1} \mathscr{H}^{N-1}\left(\partial_{\text {red }}\left\{v_{0}>0\right\} \cap B_{1}\right) .
$$

On the other hand, by non-degeneracy, as in proof of Theorem 6.1, there holds

$$
f_{B_{1 / 5}(0)} v_{0}^{\frac{1}{\alpha}} d x \geq C_{3}
$$


for a positive universal constant $C_{3}$. Finally from (9.9) and 9.10) we conclude

$$
\mathscr{H}^{N-1}\left(\partial_{\text {red }}\left\{v_{0}>0\right\} \cap B_{1}\right) \geq c_{0},
$$

for a universal constant $c_{0}$ and the estimate by below in proven. The total measure of the reduced free boundary follows now by classical considerations.

\section{Acknowledgments}

This paper is part of the first author's $\mathrm{PhD}$ thesis conducted in the Department of Mathematics at Universidade Federal do Ceará, Brazil. Both authors would like to express their gratitude to this institution for fostering such a enjoyable and productive scientific atmosphere. The authors would like to thank the anonymous referee for a careful and thoughtful revision which greatly improved the final outcome of this work. The authors also thank Cyril Imbert for a friendly and elucidating discussion on the heuristics of Ishii-Lions method. This work has been partially supported by CNPq-Brazil and Capes-Brazil.

\section{References}

[1] Alt, H. M. and Caffarelli, L. A. Existence and regularity for a minimum problem with free boundary. J. Reine Angew. Math., 325, (1981), 105-144.

[2] Alt, H. M. and Phillips, D. A free boundary problem for semilinear elliptic equations. J. Reine Angew. Math., 368, (1986), 63-107.

[3] Barles, G. A weak Bernstein method for fully nonlinear elliptic equations. Differential Integral Equations 4 (1991), no. 2, 241-262.

[4] Barles, Guy; Chasseigne, Emmanuel; Imbert, Cyril Hölder continuity of solutions of secondorder non-linear elliptic integro-differential equations. J. Eur. Math. Soc. (JEMS) 13 (2011), no. $1,1-26$.

[5] Cabre, Xavier; Caffarelli, Luis A. Interior $C^{2, \alpha}$ regularity theory for a class of nonconvex fully nonlinear elliptic equations. J. Math. Pures Appl. 82 (9) (2003), 573-612

[6] Caffarelli, L. A. The regularity of free boundaries in higher dimensions. Acta Math., 139 (1977), no. 3-4, 155-184.

[7] Caffarelli, Luis A. Interior a priori estimates for solutions of fully nonlinear equations. Ann. of Math. (2) 130 (1989), no. 1, 189-213.

[8] Caffarelli, Luis A.; Cabré, Xavier Fully nonlinear elliptic equations. American Mathematical Society Colloquium Publications, 43. American Mathematical Society, Providence, RI, 1995. 
[9] L. Caffarelli, L. Karp and H. Shahgholian, Regularity of a free boundary with application to the Pompeiu problem. Ann. Math. 151 (2000), 269-292.

[10] Crandall, Michael G.; Ishii, Hitoshi Lions, Pierre-Louis User's guide to viscosity solutions of second order partial differential equations. Bull. Amer. Math. Soc. (N.S.) 27 (1992), no. 1, $1-67$.

[11] Crandall, M. G., Rabinowitz, P. H. and Tartar, L. On a Dirichlet problem with a singular nonlinearity. Comm. Partial Differential Equations, 2 (1977), no. 2, 193-222.

[12] Evans, L. C., Classical solutions of fully nonlinear, convex, second-order elliptic equations. Comm. Pure Appl. Math. 35(3), 333-363, 1982.

[13] Felmer, P.; Quaas, A.; Sirakov, B. Existence and regularity results for fully nonlinear equations with singularities. Math. Ann. (2 November 2011), pp. 1-24. doi:10.1007/s00208-0110741-5.

[14] Giaquinta, M.; Giusti, E. Differentiability of minima of non-differentiable functionals. Invent. Math. 72, 285-298 (1983).

[15] Giaquinta, M.; Giusti, E. Sharp estimates for the derivatives of local minima of variational integrals. Bollettino U.M.I. 6, 3-A (1984) 239-248.

[16] Imbert, C.; Silvestre, L.; $C^{1, \alpha}$ regularity of solutions of some degenerate fully non-linear elliptic equations. Adv. Math. 233 (2013), 196-206.

[17] Ishii, H.; Lions, P.-L. Viscosity solutions of fully nonlinear second-order elliptic partial differential equations. J. Differential Equations 83 (1990), no. 1, 26-78.

[18] Krylov, N. V.; Safonov, M. V. An estimate of the probability that a diffusion process hits a set of positive measure. Dokl. Akad. Nauk. SSSR 245 (1979), 235-255. English translation in Soviet Math Dokl. 20 (1979), 235-255.

[19] Lee, Ki-Ahm; Shahgholian, Henrik Regularity of a free boundary for viscosity solutions of nonlinear elliptic equations. Commun. Pure Appl. Math. 54, No.1, 43-56 (2001).

[20] N. Nadirashvili and S. Vladut, Nonclassical Solutions of Fully Nonlinear Elliptic Equations II. Hessian Equations and Octonions. Geom. Funct. Anal. 21 (2011), 483-498

[21] Phillips, D. A minimization problem and the regularity of solutions in the presence of a free boundary. Indiana Univ. Math. J., 32 (1983), 1-17.

[22] Phillips, D. Hausdorff measure estimates of a free boundary for a minimum problem. Comm. Partial Differential Equations, 8 (1983), 1409-1454.

[23] Ricarte G.; Teixeira, E. Fully nonlinear singularly perturbed equations and asymptotic free boundaries. J. Funct. Anal., vol. 261, Issue 6, 2011, 1624-1673. 
[24] Silvestre, L.; Teixeira, E. Asymptotic regularity estimates for fully nonlinear equations. Preprint.

[25] Teixeira, E. V. A variational treatment for general elliptic equations of the flame propagation type: regularity of the free boundary. Ann. Inst. H. Poincaré Anal. Non Linéaire, 25, (2008), no. 4, 633-658.

[26] Teixeira, E. V. Universal moduli of continuity for solutions to fully nonlinear elliptic equations. Preprint http://arxiv.org/abs/1111.2728

[27] Weiss, G. Partial regularity for weak solutions of an elliptic free boundary problem. Comm. Partial Differential Equations, 23, (1998), no. 3-4, 439-455.

EDUARDO V. TEIXEIRA

Universidade Federal do Ceará

Campus of Pici - Bloco 914

Fortaleza - Ceará - Brazil

$60.455-760$

teixeira@mat.ufc.br
DAMIÃo ARAÚJO

Universidade Federal do Ceará

Campus of Pici - Bloco 914

Fortaleza - Ceará - Brazil

$60.455-760$

djunio@mat.ufc.com 